\title{
HI observations of blue compact galaxies from the first and second Byurakan surveys ${ }^{\star}$
}

\author{
Trinh X. Thuan ${ }^{1,3}$, V.A. Lipovetsky ${ }^{\dagger, 2}$, J.-M. Martin ${ }^{4}$, and S.A. Pustilnik ${ }^{2}$ \\ 1 Astronomy Department, University of Virginia, Charlottesville, VA 22903, U.S.A. \\ e-mail: txt@virginia.edu \\ 2 Special Astrophysical Observatory, Russian Academy of Sciences, Nizhnij Arkhyz, Karachai-Circessia 357147, Russia \\ e-mail: sap@sao.ru \\ ${ }^{3}$ Département d'Astronomie Extragalactique et de Cosmologie, Observatoire de Paris, F-92195 Meudon Cedex, France \\ 4 Département de Radioastronomie ARPEGES, Observatoire de Paris, F-92195 Meudon Cedex, France \\ e-mail: jmmartin@obspm.fr
}

Received August 11; accepted November 24, 1998

\begin{abstract}
We present the results of a neutral hydrogen survey of 79 galaxies from a statistical sample of 88 Blue Compact Galaxies (BCGs) selected from the First and Second Byurakan objective prism surveys to have a HII region-like spectrum, an equivalent width of the [O III] $\lambda 5007$ line larger than $\sim 50 \AA$, and a velocity $\leq 6000 \mathrm{~km} \mathrm{~s}^{-1}$. The detection rate for the statistical sample is $74 \%$. HI masses range between $410^{7} M_{\odot}$ and $510^{9} M_{\odot}$ with the HI mass distribution peaking at $310^{8} M_{\odot}$. The full width at half-maximum of the HI profile varies between $\sim 30 \mathrm{~km} \mathrm{~s}^{-1}$ and $160 \mathrm{~km} \mathrm{~s}^{-1}$, with a mean of $\sim 92 \mathrm{~km} \mathrm{~s}^{-1}$. These small widths are characteristic of dwarf galaxies. For comparison, we have also observed an additional 92 BCGs with weaker star formation and/or larger distances, and/or interesting astrophysical properties. These in general have larger widths and HI masses.
\end{abstract}

Key words: galaxies: compact — galaxies: ism — galaxies: startburst — radio lines: galaxies — radio lines: ISM

\section{Introduction}

Blue Compact Galaxies (BCGs) are low-luminosity $\left(M_{B} \geq-18\right)$ extragalactic objects characterized by a very high current star formation rate $(0.1-1) M_{\odot} \mathrm{yr}^{-1}$ (Fanelli et al. 1988), responsible for their optical appearance: one or several high surface brightness compact star-forming regions superimposed in the majority of cases on a more extended low surface brightness component (Loose \& Thuan 1985; Kunth et al. 1988; Papaderos

Send offprint requests to: Trinh X. Thuan

$\dagger$ Deceased 1996 September 22.

* Tables 1, 2, 3 also available in electronic form at the CDS via anonymous ftp to cdsarc.u-strasbg.fr (130.79.128.5) or via http://cdsweb.u-strasbg.fr/Abstract.html et al. 1996). Their optical spectra show strong narrow emission lines on top of a stellar continuum which is rising towards the blue, similar to spectra of HII regions, and indicating the presence of a large population of massive OB stars. Much attention has been devoted to BCGs since their discovery by Sargent \& Searle (1970), because of the realization that BCGs best approximate young galaxies, as implied by their low metallicity $\left(Z_{\odot} / 50<Z<Z_{\odot} / 3\right)$, and their very high gas content (see Thuan 1991 for a review). Because the gas in the most metal-poor BCGs has not been processed through many generations of stars, it is nearly primordial. Thus such BCGs are the best objects in which to attack such problems as the determination of the helium abundance (Izotov \& Thuan 1998 and references therein). They are also excellent nearby laboratories for studying physical processes which occurred in the galaxy formation era, in a very metal-deficient environment. Many observational studies of BCGs have been carried out in a variety of wavelength domains, from spectrophotometric studies to derive metal abundances (e.g. Thuan et al. 1995) to UV spectral synthesis (Fanelli et al. 1988) and optical and near-infrared imaging to study stellar populations (Thuan 1983), to single-dish and interferometric $21 \mathrm{~cm}$ observations to examine the $\mathrm{HI}$ content and structure (Thuan \& Martin 1981; Lequeux \& Viallefond 1980; Viallefond \& Thuan 1983). In this paper, we present a large set of HI single dish observations for a new sample of BCGs assembled from the First (Markarian et al. 1989) and Second Byurakan (Markarian et al. 1983) objective prism surveys. Such observations are crucial for the understanding of BCGs for several reasons:

1) They yield accurate $\left( \pm 15 \mathrm{~km} \mathrm{~s}^{-1}\right)$ redshifts for the study of the large scale distribution of BCGs (Pustilnik et al. 1995). 
2) The HI flux allows to determine the neutral atomic gas mass and the width of the HI profile yields an estimate of the total mass of the BCG. The gas mass fraction combined with abundance measurements are crucial for testing chemical evolution models of BCGs, such as closed-box models, models with galactic winds or infall, etc. (Lequeux et al. 1979; Pilyugin 1993; Marconi et al. 1994; Peimbert et al. 1994; Carigi et al. 1995; Pustilnik et al. 1996).

3) Combining with other data, the HI data permit to derive global parameters of BCGs (such as $M(\mathrm{HI}) / L_{B}$ or $M$ (total) $/ L_{B}$, study general trends and correlations (for example $M(\mathrm{HI}) / L_{B}$ vs. $L_{B}$ ) suggested by various galaxy formation theories and compare with other types of galaxies. For example, one question of great interest is the relationship between BCGs and another class of dwarf galaxies, the low-surface-brightness (LSB) dwarfs. Star formation in BCGs is known to occur in bursts lasting $\leq 10^{8} \mathrm{yr}$, separated by long quiescent periods of $2-310^{9}$ yr (Thuan 1991). If LSB dwarfs are BCGs in their quiescent phases, then their HI properties should be statistically similar (Thuan 1985). The HI data obtained here can be compared statistically with the large HI data set for LSB dwarfs assembled by Schneider, Thuan and their colleagues $(1990,1992)$ to test the above hypothesis. Finally, a HI single-dish survey allows to judge the feasibility of follow-up HI interferometric studies of particularly interesting objects in the sample.

The first comprehensive HI survey of BCGs was carried out by Thuan \& Martin (1981). These authors assembled a list of 115 blue compact dwarfs known at that time from the objective prism surveys of Markarian and Haro, with a few objects from Zwicky and other investigators. Other HI surveys of BCGs followed, such as that of Gordon \& Gottesman (1981) which included mainly brighter BCGs $\left(M_{B}<-18\right)$ from the Markarian, Haro and Zwicky lists, that of Hoffman et al. (1992) for BCGs in the Virgo cluster, and that of Staveley-Smith et al. (1992) for a small sample of nearby BCGs.

The BCG sample we are concerned with here was primarily assembled from objective prism survey plates obtained with the $1 \mathrm{~m}$ Schmidt Telescope at the Byurakan Observatory during the Second Byurakan Survey (SBS; Markarian et al. 1983). The objective prism plates cover the sky area defined by $7^{\mathrm{h}} 40^{\mathrm{m}} \leq \alpha \leq 17^{\mathrm{h}} 20^{\mathrm{m}}, 49^{\circ} \leq$ $\delta \leq 61^{\circ}$, an area of about 1000 square degrees. All objects with prism spectra showing strong or moderate emission lines were observed spectroscopically with the $6 \mathrm{~m}$ telescope of the Special Astrophysical Observatory. Then all emission-line galaxies with a HII region-like spectrum and an equivalent width of the [O III $] \lambda 5007$ emission line larger than $\approx 30 \AA$, were selected to constitute the BCG sample. The above criterion excluded massive nuclear starburst galaxies and galaxies with an active galactic nucleus such as Seyfert galaxies and quasars. In addition, we have also added $\sim 50$ BCGs from the First Byurakan Survey
(Markarian et al. 1989) and a few BCGs from the Case Survey (Pesch \& Sanduleak 1987) in the same sky area and satisfying the same selection criterion. The resulting sample contains a total of 220 BCGs (Izotov et al. 1993). The redshift distribution of the total sample along with the large-scale space distribution are given in Pustilnik et al. (1995). The BCG sample is reasonably complete out to $\approx 10000 \mathrm{~km} \mathrm{~s}^{-1}$, with increasing incompleteness beyond. The redshift distribution shows 3 peaks, one at $V \approx 1000$ $\mathrm{km} \mathrm{s}^{-1}$ due to the Virgo cluster, and two additional peaks at $V \approx 3000 \mathrm{~km} \mathrm{~s}^{-1}$ and $V \approx 7000-10000 \mathrm{~km} \mathrm{~s}^{-1}$. In order not to spend inordinately large amounts of telescope time on a single galaxy and still reach interesting upper limits for the BCG HI content with a given telescope sensitivity, we have extracted a complete subsample of 88 BCGs by further imposing a lower limit on the equivalent width of the $[\mathrm{O}$ III $] \lambda 5007$ emission line of $50 \AA$, and a velocity upper limit of $6000 \mathrm{~km} \mathrm{~s}^{-1}$.

Of these 88 BCGs, 10 were already observed in earlier studies (Thuan \& Martin 1981; Gordon \& Gottesman 1981). We have obtained new HI observations for 77 BCGs, and obtained measurements with better signal-tonoise ratio of two BCGs with published data. One BCG which is close to a HI rich galaxy with the same velocity was not observed.

This complete subsample will be used for statistical studies in a subsequent paper. For comparison, we have observed in addition 20 BCGs in the SBS zone with $V \leq$ $6000 \mathrm{~km} \mathrm{~s}^{-1}$ but with less strong emission lines, 47 BCGs not in the SBS zone with the same velocity limit, and 17 BCGs in the SBS zone with $6000 \leq V \leq 14000 \mathrm{~km} \mathrm{~s}^{-1}$, which are of particular astrophysical interest. Finally 8 more BCGs outside the SBS zone with $V \geq 6000 \mathrm{~km} \mathrm{~s}^{-1}$ were observed. Altogether we have obtained HI parameters or upper limits for 171 BCGs.

We discuss the HI observations and the data reduction in Sect. 2. In Sect. 3 we describe the data tables and present the observed HI profiles. We give a preliminary discussion of the data in Sect. 4. A more complete discussion is deferred to a subsequent paper.

\section{Observations and data reduction}

The $21 \mathrm{~cm}$ observations were carried out in 1992-1996 with the Nançay ${ }^{1} 300-\mathrm{m}$ and Green Bank ${ }^{2}$ 43-m radio telescopes.

The Nançay radio telescope has a half-power beam width $(\mathrm{HPBW})$ of $3.7^{\prime}(\mathrm{EW}) \times 22^{\prime}(\mathrm{NS})$ at the

\footnotetext{
1 The Nançay Radioastronomy Station is part of the Paris Observatory and is operated by the Ministère de l'Éducation Nationale and Institut des Sciences de l'Univers of the Centre National de la Recherche Scientifique.

2 The Green Bank Observatory is part of the National Radio Astronomy Observatory, which is operated by Associated Universities, Inc. for the National Science Foundation.
} 
declination $\delta=0^{\circ}$. We used a dual-polarization receiver, with a system temperature of $\approx 40 \mathrm{~K}$ in the horizontal linear polarization and $\approx 40 \mathrm{~K}$ and $60 \mathrm{~K}$ in the vertical linear polarization in the periods 1992-1994 and 1995 respectively. As all the candidate BCGs had known optical redshifts from the $6 \mathrm{~m}$ spectroscopic observations, we split the 1024-channel autocorrelator into two, covering a bandwidth of $6.4 \mathrm{MHz}$ each, and centered at the frequency corresponding to the optical redshift. In this configuration, each segment covered a velocity range of $1350 \mathrm{~km} \mathrm{~s}^{-1}$. The channel spacing was $2.6 \mathrm{~km} \mathrm{~s}^{-1}$ before smoothing and the effective resolution after averaging pairs of adjacent channels and Hanning smoothing was $\approx 21 \mathrm{~km} \mathrm{~s}^{-1}$. The gain of the telescope was $1.1 \mathrm{~K} / \mathrm{Jy}$ at the declination $\delta=0^{\circ}$. The observations were made in the standard total power (position switching) mode with 2-minute on-source and 2 minute off source integrations. Typically, we aim to achieve a rms noise of $2.5 \mathrm{mJy}$ per channel, after smoothing the channel spacing to $21 \mathrm{~km}$ $\mathrm{s}^{-1}$. This leads to a typical integration time of 1 hour on the galaxy and 1 hour on the comparizon field. For BCGs with the faintest HI flux densities, the ON integration time may go up to 3-4 hours. Since the calibration noise diode power has changed significantly over the years, and since its power is frequency-dependent, we have exercised great care in improving the standard Nançay radio telescope calibration method to correct the data for the time and frequency dependencies. This procedure is detailed in Appendix A.

The NRAO $43 \mathrm{~m}$ radio telescope has a HPBW of $22^{\prime}$. We use a two channel dual-polarization prime-focus receiver with a system temperature of $\approx 20 \mathrm{~K}$ in both polarizations. A bandwidth of $20 \mathrm{MHz}$ covering $4225 \mathrm{~km} \mathrm{~s}^{-1}$ was used with the 1024-channel autocorrelator split in two. The channel spacing was $\approx 8 \mathrm{~km} \mathrm{~s}^{-1}$ and the effective resolution after Hanning smoothing was $\approx 16 \mathrm{~km} \mathrm{~s}^{-1}$. The observations were also made in the position switching mode, with 6 minutes off-source and 6 minutes on-source integrations. The calibration signal levels and telescope pointing errors were checked by observing standard calibrators listed in the $43 \mathrm{~m}$ Telescope Observer's Manual. The calibration noise diode temperature was measured in the laboratory before the observing run. The gain of the telescope was $0.29 \mathrm{~K} / \mathrm{Jy}$.

The Green Bank data was reduced using NRAO's standard LINE program, while the Nançay data was reduced using the software developed by the telescope's staff. For each integration, the two polarizations which were detected independently were averaged to improve sensitivity. The baselines were generally well fitted by a polynomial of third order or lower and subtracted out.

To check that the data taken from the two telescopes are on the same flux scale, we have observed several galaxies with both telescopes. To within the errors, the Nançay and Green Bank fluxes agree with each other.

\section{Results}

We present the data in three tables. Table 1 gives the data for the galaxies for which we feel the HI results are secure. This means we are relatively certain that the signal is not produced by confusion or receiver baseline anomalies, and that the signal-to-noise value is adequate. Table 2 , on the other hand, lists detections of emission which may be all right, but for various reasons such as confusion or a low signal-to-noise ratio, we feel should be used more cautiously. Table 3 lists the undetected galaxies and upper limits for their HI flux densities.

The cut-off between Table 1 and Table 2 is not rigorously defined as it is based on a mixture of subjective evaluation of the HI profiles as well as more objective guidelines. The possibility of confusion was checked for every galaxy by using the Lyon-Meudon Extragalactic Database (LEDA) to generate sky plots of a circular area of radius $30^{\prime}$ centered on the galaxy. This allows also to check for the environment of the galaxy, whether it is isolated or in a group. The properties and angular separations of possible companion galaxies are given in the Notes to Tables 1 and 2. There is generally a signal-tonoise ratio lower limit of 4 for inclusion in Table 1 . The signal is measured as the peak flux in the galaxy's HI profile, while the noise is measured in the baseline away from the observed signal. In a few cases, repeated observations yielded essentially the same parameters, and although the signal-to-noise ratio remained low, we have included the galaxies in Table 1.

Tables 1 and 2 include all of the basic information about the galaxies and the observations, along with the optical angular size information used in calculating corrections to the measured HI fluxes. They are organized as follows:

Column (1). Name following the IAU nomenclature. Some BCGs in physical pairs are characterized by additional letters $(\mathrm{A}, \mathrm{B}, \mathrm{N}=$ North, $\mathrm{E}=$ East), but in the tables the two components are lumped together in the same name entry, as they are not resolved by the radio telescope beam. The BCGs which are in the statistical sample are marked by a cross in all tables. Galaxies marked by an asterisk in Table 2 are non-confused.

Column (2). Cross-references to other names in the Markarian (MK, Markarian et al. 1989), Michigan (UM, Salzer et al. 1989) or in the Case (CG, Pesch et al. 1991) lists. When the galaxy is from the Second Byurakan Survey (Markarian et al. 1983; Stepanian et al. 1993a-d), it is designated by the initials SBS.

Columns (3) and (4). Right ascension and declination at the epoch 1950 . The coordinates have been measured either from the Palomar Sky Survey prints or plates, or taken to be the pointing coordinates of the $6 \mathrm{~m}$ telescope during the spectroscopic observations. The errors in each coordinate are typically between 1 and 5 arcsec. 


\begin{tabular}{|c|c|c|c|c|c|c|c|c|c|c|c|c|c|}
\hline \multirow[t]{2}{*}{ IAAU name } & \multirow{3}{*}{$\begin{array}{c}\text { Other } \\
\text { name or } \\
\text { prefix } \\
2\end{array}$} & \multicolumn{2}{|c|}{ "Coord. (1950.0) } & \multirow{2}{*}{$\begin{array}{c}\text { Angul. } \\
\text { size } \\
\text { axb }\end{array}$} & \multirow[t]{2}{*}{ 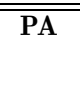 } & \multirow{2}{*}{$\begin{array}{l}V(\mathbf{H I}) \\
\mathrm{km} \mathrm{s}^{-1}\end{array}$} & \multirow{2}{*}{$\begin{array}{l}\text { Dist. } \\
\text { Mpc } \\
\text { Vir }\end{array}$} & \multirow{2}{*}{$\begin{array}{c}W_{50} \\
\mathbf{k m ~ s}^{-1}\end{array}$} & \multirow{2}{*}{$\begin{array}{c}W_{20} \\
\mathbf{k m ~ s}^{-1}\end{array}$} & \multirow{2}{*}{$\begin{array}{l}\text { Obs. Flux } \\
\text { Jy km s s }^{-1}\end{array}$} & \multirow{2}{*}{$\begin{array}{l}\text { Cor. Flux } \\
\text { Jy km s }^{-1}\end{array}$} & \multirow{2}{*}{$\begin{array}{c}\text { Log } \\
M(\mathbf{H I}) / \\
M_{\odot}\end{array}$} & \multirow{2}{*}{$\begin{array}{l}R \\
\& \\
N\end{array}$} \\
\hline & & $\begin{array}{l}\text { R.A. } \\
\text { h m s }\end{array}$ & $\begin{array}{l}\text { Dec. } \\
0,11\end{array}$ & & & & & & & & & & \\
\hline 1 & & 3 & 4 & 5 & 6 & 7 & 8 & 9 & 10 & 11 & 12 & 13 & 14 \\
\hline $0012-018$ & MK546 & 001201.6 & 014943 & $30 \times 27$ & 30 & $3974 \pm 17$ & 52.7 & $119 \pm 29$ & $156 \pm 40$ & $0.62 \pm 0.15$ & $0.63 \pm \mathbf{0 . 1 5}$ & 8.62 & $\mathrm{N93}^{*}$ \\
\hline $0034+356$ & MK953 & 003430.8 & 353739 & $44 \times 16^{\mathrm{e}}$ & -10 & $4441 \pm 1$ & 61.3 & $81 \pm 21$ & $147 \pm 33$ & $1.96 \pm \mathbf{0 . 2 4}$ & $1.97 \pm \mathbf{0 . 2 4}$ & 9.24 & N94* \\
\hline $0111+075$ & MK564 & 011112.1 & 073107 & $35 \times 25^{\mathrm{e}}$ & 45 & $5514 \pm 13$ & 73.3 & $149 \pm 26$ & $171 \pm 41$ & $0.90 \pm \mathbf{0 . 2 2}$ & $0.92 \pm 0.22$ & 9.07 & N94* \\
\hline $0125-065$ & MK996 & 012504.6 & -063507 & $62 \times 50^{\mathrm{e}}$ & 30 & $1622 \pm 21$ & 21.7 & $83 \pm 42$ & $177 \pm 65$ & $1.26 \pm 0.20$ & $1.30 \pm \mathbf{0 . 2 0}$ & 8.16 & N94* \\
\hline $0142+046$ & UM133 & 014205.3 & 043825 & & & $1623 \pm 04$ & 22.4 & $104 \pm 07$ & $122 \pm 11$ & $4.30 \pm 0.67$ & & 8.71 & G92* \\
\hline $0143+346$ & MK1006 & $0143 \quad 33.4$ & 344040 & $58 \times 36^{\mathrm{e}}$ & 20 & $5508 \pm 05$ & 75.0 & $129 \pm 29$ & $152 \pm 14$ & $1.99 \pm 0.20$ & $2.02 \pm 0.20$ & 9.43 & N94* \\
\hline $0144+024 \mathrm{~A}$ & UM369 & 014429.0 & 022638 & $37 \times 17^{\mathrm{e}}$ & 45 & $5694 \pm 08$ & 75.2 & $112 \pm 15$ & $128 \pm 24$ & $0.67 \pm 0.19$ & $0.68 \pm 0.19$ & 8.96 & N94* \\
\hline $0214+292$ & MK1030 & 021439.2 & 291727 & & & $5262 \pm 03$ & 71.3 & $72 \pm 07$ & $100 \pm 10$ & $6.65 \pm 0.72$ & & 9.90 & G92* \\
\hline $0216-032$ & MK1031 & 021620.3 & -031614 & & & $2360 \pm 12$ & 31.2 & $154 \pm 24$ & $182 \pm 38$ & $2.63 \pm 0.80$ & & 8.78 & G92 \\
\hline $0225-103$ & MK1039 & 022506.2 & -102321 & $117 \times 43^{\mathrm{e}}$ & 90 & $2098 \pm 05$ & 27.3 & $149 \pm 11$ & $198 \pm 17$ & $5.96 \pm \mathbf{0 . 3 6}$ & $6.76 \pm \mathbf{0 . 4 0}$ & 9.08 & N94* \\
\hline $0225-104$ & MK1042 & 022538.0 & -102423 & $32 \times 20^{\mathrm{e}}$ & 15 & $2133 \pm 08$ & 27.8 & $83 \pm 16$ & $117 \pm 26$ & $1.32 \pm 0.22$ & $1.33 \pm 0.22$ & 8.38 & N94* \\
\hline $0237+166$ & MK1182 & 023754.8 & 163700 & $47 \times 32$ & 0 & $8754 \pm 17$ & 116.3 & $176 \pm 35$ & $225 \pm 55$ & $1.86 \pm \mathbf{0 . 3 6}$ & $1.88 \pm \mathbf{0 . 3 6}$ & 9.78 & $\mathrm{N93}$ \\
\hline $0305-140$ & MK1069 & 030557.5 & -140535 & $60 \times 25$ & 60 & $1562 \pm 03$ & 20.2 & $106 \pm 05$ & $136 \pm 08$ & $8.50 \pm 0.46$ & $8.74 \pm \mathbf{0 . 4 8}$ & 8.93 & N93 \\
\hline $0335-052$ & SBS & 033515.1 & -051226 & $14 \times 12$ & 0 & $4043 \pm 05$ & 52.8 & $83 \pm 09$ & $105 \pm 14$ & $1.28 \pm 0.22$ & $1.45 \pm \mathbf{0 . 2 5}$ & 8.98 & N93* \\
\hline $0741+535^{+}$ & MK1409 & 074134.8 & 533255 & $65 \times 21^{\mathrm{e}}$ & -45 & $5564 \pm 30$ & 77.1 & $109 \pm 60$ & $216 \pm 94$ & $1.89 \pm 0.34$ & $1.93 \pm \mathbf{0 . 3 5}$ & 9.43 & N94* \\
\hline $0743+550$ & MK1410 & 074310.0 & 550415 & $45^{\mathrm{e}}$ & -25 & $5490 \pm 04$ & 76.2 & $167 \pm 08$ & $183 \pm 12$ & $3.29 \pm 0.37$ & $3.32 \pm 0.38$ & 9.66 & N94* \\
\hline $0749+568^{+}$ & SBS & 074937.8 & 564941 & $25 \times 25^{\mathrm{e}}$ & 30 & $5488 \pm 25$ & 76.3 & $91 \pm 49$ & $150 \pm 77$ & $0.58 \pm 0.17$ & $0.58 \pm \mathbf{0 . 1 7}$ & 8.90 & N94* \\
\hline $0813+521$ & SBS & 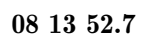 & 521156 & $27 \times 27$ & 0 & $7065 \pm 16$ & 96.8 & $79 \pm 32$ & $119 \pm 50$ & $0.83 \pm \mathbf{0 . 2 6}$ & $0.84 \pm \mathbf{0 . 2 6}$ & 9.27 & N93 \\
\hline $0832+664$ & MK93 & $\begin{array}{lll}08 & 32 & 09.7\end{array}$ & 662423 & $26 \times 13^{\mathrm{e}}$ & 45 & $5292 \pm 05$ & 74.2 & $252 \pm 10$ & $276 \pm 15$ & $2.72 \pm \mathbf{0 . 2 3}$ & $2.79 \pm 0.23$ & 9.56 & N94 \\
\hline $0847+612$ & MК99 & 084725.2 & 611229 & $42 \times 23$ & 60 & $4120 \pm 12$ & 58.8 & $126 \pm 23$ & $179 \pm 37$ & $2.18 \pm 0.29$ & $2.21 \pm 0.29$ & 9.26 & N95* \\
\hline $0916+543$ & SBS & 091639.1 & 541911 & $36 \times 26$ & -60 & $3582 \pm 18$ & 51.6 & $126 \pm 36$ & $179 \pm 56$ & $0.75 \pm 0.20$ & $0.76 \pm 0.20$ & 8.68 & N93 \\
\hline $0917+527^{+}$ & MK1416 & $\begin{array}{llll}09 & 17 & 25.7\end{array}$ & 524653 & $30 \times 15$ & -30 & $2323 \pm 07$ & 35.4 & $65 \pm 13$ & $99 \pm 21$ & $1.96 \pm 0.32$ & $1.97 \pm 0.32$ & 8.77 & N94* \\
\hline $0930+554^{+}$ & MK116 & 093030.2 & $55 \quad 2747$ & $26 \times 13^{\mathrm{e}}$ & -40 & $751 \pm 02$ & 14.2 & $53 \pm 04$ & $84 \pm 06$ & $2.71 \pm \mathbf{0 . 1 4}$ & $2.72 \pm 0.14$ & 8.11 & N95* \\
\hline $0939+592$ & MK1423 & $\begin{array}{ll}09 & 3924.4\end{array}$ & 591208 & $105 \times 60$ & -45 & $1358 \pm 08$ & 23.1 & $95 \pm 15$ & $116 \pm 24$ & $0.79 \pm 0.20$ & $0.84 \pm \mathbf{0 . 2 1}$ & 8.02 & N94* \\
\hline $0940+662$ & MK119 & 094010.7 & $6612 \quad 25$ & & & $3316 \pm 17$ & 48.6 & $158 \pm 33$ & $189 \pm 52$ & $2.86 \pm 1.24$ & & 9.20 & G92* \\
\hline $0940+544^{+}$ & SBS & 094050.8 & 542518 & & & $1638 \pm 05$ & 26.7 & $120 \pm 09$ & $130 \pm 15$ & $3.06 \pm 0.97$ & & 8.71 & G91* \\
\hline $0942+573^{+}$ & MK1424 & 094256.4 & 572055 & $60 \times 30$ & -30 & $1177 \pm 19$ & 20.6 & $127 \pm 39$ & $190 \pm 60$ & $2.46 \pm 0.37$ & $2.50 \pm 0.38$ & 8.40 & N94* \\
\hline $0946+487$ & MK1426 & 094603.1 & $48 \quad 4749$ & & & $1835 \pm 04$ & 29.1 & $82 \pm 07$ & $92 \pm 10$ & $1.81 \pm \mathbf{0 . 5 5}$ & & 8.56 & G92* \\
\hline $0946+558^{+}$ & MK22 & 094603.4 & 554849 & & & $1551 \pm 12$ & 25.6 & $80 \pm 24$ & $101 \pm 38$ & $2.19 \pm 0.90$ & & 8.53 & G92* \\
\hline $1001+555^{+}$ & SBS & 100120.7 & $\begin{array}{lll}5533 & 18\end{array}$ & & & $1116 \pm 04$ & 19.7 & $106 \pm 08$ & $125 \pm 12$ & $4.54 \pm \mathbf{0 . 7 3}$ & & 8.62 & G92* \\
\hline $1006+578$ & SBS & 100612.1 & 574845 & $40 \times 21$ & 25 & $1512 \pm 01$ & 25.4 & $127 \pm 02$ & $137 \pm 03$ & $3.14 \pm 0.18$ & $3.16 \pm 0.18$ & 8.68 & N96* \\
\hline $1033+531^{+}$ & SBS & 103330.6 & 530634 & & & $986 \pm 04$ & 17.9 & $124 \pm 09$ & $135 \pm 14$ & $3.17 \pm 0.81$ & & 8.38 & G91 \\
\hline $1037+494^{+}$ & SBS & 103742.1 & 492805 & & & $1496 \pm 11$ & 25.0 & $117 \pm 21$ & $142 \pm 33$ & $3.47 \pm 0.95$ & & 8.71 & G91 \\
\hline $1114+587^{+}$ & SBS & 111410.7 & 584722 & $32 \times 29$ & 20 & $1594 \pm 21$ & 26.7 & $144 \pm 43$ & $200 \pm 67$ & $1.15 \pm 0.36$ & $1.16 \pm 0.36$ & 8.29 & N94* \\
\hline $1119+586^{+}$ & SBS & 111945.7 & 583605 & $20 \times 10^{\mathrm{e}}$ & 0 & $1583 \pm 06$ & 26.5 & $52 \pm 13$ & $95 \pm 20$ & $1.32 \pm 0.36$ & $1.32 \pm \mathbf{0 . 3 6}$ & 8.34 & N94* \\
\hline $1123+644$ & MK170 & 112355.8 & 642448 & & & $988 \pm 14$ & 18.4 & $90 \pm 28$ & $153 \pm 44$ & $7.52 \pm 1.24$ & & 8.78 & G92* \\
\hline $1124+541^{+}$ & MK1446 & 112445.5 & 541125 & $44 \times 39$ & 20 & $2940 \pm 11$ & 44.0 & $53 \pm 22$ & $106 \pm 34$ & $0.99 \pm 0.20$ & $1.01 \pm 0.20$ & 8.66 & N95 \\
\hline $1152+579^{+}$ & MK193 & 115251.9 & 575634 & $22 \times 19$ & -15 & $5157 \pm 17$ & 72.6 & $53 \pm 34$ & $106 \pm 54$ & $0.72 \pm \mathbf{0 . 2 2}$ & $0.72 \pm \mathbf{0 . 2 2}$ & 8.95 & N94 \\
\hline $1203+592^{+}$ & SBS & 120343.2 & 591459 & $48 \times 26$ & 5 & $3256 \pm 07$ & 48.1 & $156 \pm 13$ & $179 \pm 20$ & $4.05 \pm 0.60$ & $4.08 \pm 0.60$ & 9.35 & N94 \\
\hline $1205+557^{+}$ & SBS & 120557.5 & 554208 & $24 \times 19$ & -40 & $1740 \pm 08$ & 28.7 & $42 \pm 16$ & $63 \pm 25$ & $0.37 \pm 0.25$ & $0.37 \pm 0.25$ & 7.86 & N94 \\
\hline $1211+540^{+}$ & SBS & 121134.0 & 540158 & $23 \times 14$ & -50 & $907 \pm 05$ & 17.2 & $53 \pm 01$ & $84 \pm 16$ & $0.71 \pm 0.12$ & $0.71 \pm 0.12$ & 7.70 & N95* \\
\hline $1213+597^{+}$ & MK1468 & 121334.8 & 594709 & $46 \times 31$ & 10 & $4420 \pm 10$ & 62.7 & $253 \pm 21$ & $274 \pm 33$ & $0.96 \pm 0.25$ & $0.97 \pm 0.25$ & 8.95 & N93* \\
\hline $1241+549^{+}$ & SBS & 124130.3 & 545511 & $28 \times 15$ & 60 & $4945 \pm 13$ & 67.5 & $116 \pm 25$ & $169 \pm 40$ & $1.72 \pm 0.26$ & $1.73 \pm 0.26$ & 9.27 & N95 \\
\hline $1317+523 \mathrm{~A}^{+}$ & SBS & 131742.9 & 521957 & $35 \times 26$ & 60 & $4619 \pm 10$ & 65.8 & $137 \pm 19$ & $211 \pm 30$ & $4.74 \pm \mathbf{0 . 3 5}$ & $4.79 \pm 0.35$ & 9.69 & N96* \\
\hline $1318+5^{-} 0^{+}$ & SBS & 131807.6 & 520137 & $43 \times 28$ & 40 & $4740 \pm 09$ & 67.4 & $137 \pm 18$ & $190 \pm 29$ & $1.78 \pm 0.22$ & $1.80 \pm 0.22$ & 9.29 & N94* \\
\hline $1323+483$ & SBS,HS & 132313.1 & 481801 & $26 \times 18$ & 0 & $4925 \pm 10$ & 69.6 & $42 \pm 20$ & $63 \pm 32$ & $0.24 \pm 0.12$ & $0.24 \pm 0.12$ & 8.44 & N94* \\
\hline
\end{tabular}




\begin{tabular}{|c|c|c|c|c|c|c|c|c|c|c|c|c|c|}
\hline \multirow[t]{2}{*}{ IAU name } & \multirow{2}{*}{$\begin{array}{c}\text { Other } \\
\text { name or } \\
\text { prefix }\end{array}$} & \multicolumn{2}{|c|}{ Coord. (1950.0) } & \multirow{2}{*}{$\begin{array}{c}\text { Angul. } \\
\text { size } \\
\text { axb }\end{array}$} & \multirow[t]{2}{*}{ 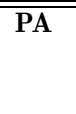 } & \multirow{2}{*}{$\begin{array}{l}V(\mathbf{H I}) \\
\mathrm{km} \mathrm{s}^{-1}\end{array}$} & \multirow{2}{*}{$\begin{array}{l}\text { Dist. } \\
\text { Mpc } \\
\text { Vir }\end{array}$} & \multirow{2}{*}{$\begin{array}{c}W_{50} \\
\mathbf{k m ~ s}^{-1}\end{array}$} & \multirow{2}{*}{$\begin{array}{l}W_{20} \\
\mathbf{k m ~ s}^{-1}\end{array}$} & \multirow{2}{*}{$\begin{array}{l}\text { Obs. Flux } \\
\text { Jy km s }^{-1}\end{array}$} & \multirow{2}{*}{$\begin{array}{c}\text { Cor. Flux } \\
\text { Jy km s }^{-1}\end{array}$} & \multirow{2}{*}{$\begin{array}{c}\text { Log } \\
M(\mathbf{H I}) / \\
M_{\odot}\end{array}$} & \multirow{2}{*}{$\begin{array}{l}R \\
\& \\
N\end{array}$} \\
\hline & & $\begin{array}{l}\text { R.A. } \\
\text { h m s }\end{array}$ & Dec. & & & & & & & & & & \\
\hline 1 & 2 & 3 & 4 & 5 & 6 & 7 & 8 & 9 & 10 & 11 & 12 & 13 & 14 \\
\hline $1331+493^{+}$ & SBS & 133119.9 & 492132 & $71 \times 58$ & 0 & $590 \pm 02$ & 11.3 & $\mathbf{5 3} \pm \mathbf{0 3}$ & $74 \pm 04$ & $6.27 \pm 0.43$ & $6.48 \pm 0.44$ & 8.29 & N93 \\
\hline $1332+518$ & MK1479 & 133242.6 & 515211 & $180 \times 120$ & $\mathbf{0}$ & $235 \pm 02$ & 4.7 & $32 \pm 04$ & $53 \pm 06$ & $4.98 \pm \mathbf{0 . 5 3}$ & $5.77 \pm \mathbf{0 . 6 1}$ & 7.48 & N96* \\
\hline $1341+594^{+}$ & SBS & 134145.0 & $\begin{array}{ll}592507\end{array}$ & $23 \times 15$ & 60 & $3037 \pm 07$ & 45.6 & $53 \pm 14$ & $84 \pm 22$ & $0.68 \pm \mathbf{0 . 0 6}$ & $0.68 \pm 0.06$ & 8.52 & N94 \\
\hline $1358+554 E^{+}$ & SBS & 135848.8 & 552944 & $33 \times 23$ & 75 & $3857 \pm 05$ & 55.9 & $63 \pm 09$ & $84 \pm 15$ & $1.41 \pm 0.31$ & $1.43 \pm 0.31$ & 9.02 & N95-96* \\
\hline $1400+461$ & CG328 & 140011.6 & 460610 & $47 \times 35$ & -15 & $2095 \pm 04$ & 33.4 & $123 \pm 07$ & $134 \pm 11$ & $1.44 \pm \mathbf{0 . 3 6}$ & $1.46 \pm \mathbf{0 . 3 6}$ & 8.58 & N93 \\
\hline $1413+495^{+}$ & CG383 & 141318.8 & 493426 & $37 \times 21$ & 60 & $3923 \pm 11$ & 56.8 & $116 \pm 22$ & $148 \pm 34$ & $1.18 \pm 0.27$ & $1.19 \pm 0.27$ & 8.96 & N95 \\
\hline $1415+437$ & CG389 & 141503.2 & 434355 & $81 \times 30$ & 30 & $609 \pm 02$ & 11.4 & $53 \pm \mathbf{0 3}$ & $74 \pm 05$ & $4.73 \pm \mathbf{0 . 3 3}$ & $4.85 \pm \mathbf{0 . 3 4}$ & 8.17 & N95 \\
\hline $1422+573$ & MK812 & 142257.8 & 572148 & & & $3171 \pm 07$ & 47.2 & $169 \pm 14$ & $190 \pm 22$ & $2.99 \pm 0.57$ & & 9.20 & G92* \\
\hline $1430+596^{+}$ & SBS & 143003.5 & 593642 & $53 \times 16$ & -40 & $1855 \pm 20$ & 30.4 & $169 \pm 24$ & $211 \pm 37$ & $1.59 \pm 0.28$ & $1.61 \pm 0.29$ & 8.54 & N94* \\
\hline $1430+526 \mathrm{~A}^{+}$ & CG470 & 143053.3 & 523759 & $60 \times 30^{\mathrm{e}}$ & -30 & $3424 \pm 06$ & 50.4 & $84 \pm 12$ & $116 \pm 18$ & $1.58 \pm 0.23$ & $1.61 \pm 0.23$ & 8.98 & N95* \\
\hline $1437+370$ & MK475 & 143703.7 & 370113 & $39 \times 26$ & 70 & $583 \pm 02$ & 9.5 & $32 \pm 05$ & $42 \pm 08$ & $0.15 \pm 0.04$ & $0.15 \pm 0.04$ & 6.50 & N95 \\
\hline $1448+526^{+}$ & MK826 & 144827.6 & 523643 & & & $726 \pm 06$ & 14.9 & $69 \pm 11$ & $85 \pm 17$ & $1.09 \pm 0.50$ & & 7.76 & G92 \\
\hline $1455+443$ & CG587 & 145532.9 & 442237 & & & $3563 \pm 17$ & 52.0 & $132 \pm 34$ & $171 \pm 53$ & $1.97 \pm \mathbf{0 . 6 9}$ & & 9.10 & G92 \\
\hline $1504+514 \mathrm{~N}^{+}$ & CG625 & $\begin{array}{ll}1504 & 18.8\end{array}$ & 512654 & $60 \times 53$ & -10 & $3796 \pm 04$ & 55.1 & $148 \pm 07$ & $179 \pm 11$ & $5.54 \pm 0.32$ & $5.70 \pm \mathbf{0 . 3 3}$ & 9.61 & N94* \\
\hline $1506+553$ & CG638 & 150625.0 & 552235 & $125 \times 90$ & 70 & $3367 \pm 08$ & 49.7 & $102 \pm 15$ & $146 \pm 24$ & $4.81 \pm 0.37$ & $5.49 \pm 0.42$ & 9.51 & N94* \\
\hline $1509+527$ & CG652 & $\begin{array}{lll}15 & 09 & 31.8\end{array}$ & 524446 & $33 \times 25^{\mathrm{e}}$ & & $3545 \pm 13$ & 51.9 & $126 \pm 25$ & $157 \pm 41$ & $0.65 \pm 0.16$ & $0.66 \pm 0.16$ & 8.62 & N94* \\
\hline $1519+496$ & CG690 & $\begin{array}{lll}15 & 19 & 16.8\end{array}$ & 494134 & $33 \times 21$ & 90 & $4543 \pm 04$ & 64.9 & $194 \pm 08$ & $206 \pm 13$ & $0.96 \pm \mathbf{0 . 2 3}$ & $0.97 \pm \mathbf{0 . 2 3}$ & 8.98 & N94* \\
\hline $1524+554^{+}$ & SBS & 152440.0 & 552530 & $41 \times 24$ & 45 & $3409 \pm 04$ & 50.3 & $190 \pm 07$ & $211 \pm 11$ & $2.43 \pm 0.21$ & $2.46 \pm 0.21$ & 9.16 & N93 \\
\hline $1535+554^{+}$ & MK487 & 153548.8 & 552535 & $43 \times 39$ & -65 & $667 \pm 10$ & 50.3 & $74 \pm 20$ & $116 \pm 31$ & $1.41 \pm 0.26$ & $1.44 \pm 0.26$ & 8.93 & N96 \\
\hline $1551+601 \mathrm{~A}^{+}$ & SBS & 155146.3 & 601129 & $45 \times 24$ & 50 & $2932 \pm 10$ & 44.2 & $63 \pm 20$ & $95 \pm 31$ & $1.05 \pm 0.27$ & $1.06 \pm 0.27$ & 8.69 & N94* \\
\hline $1629+205$ & MK884 & 162938.2 & 203101 & $30 \times 25$ & 90 & $5151 \pm 04$ & 71.6 & $137 \pm 08$ & $158 \pm 13$ & $1.45 \pm 0.19$ & $1.46 \pm \mathbf{0 . 1 9}$ & 9.25 & N95 \\
\hline $1634+523^{+}$ & MK1499 & 163407.8 & 521857 & $39 \times 34$ & 40 & $2700 \pm 04$ & 41.0 & $84 \pm 40$ & $137 \pm 62$ & $0.44 \pm 0.11$ & $0.44 \pm 0.11$ & 8.24 & N96 \\
\hline $1640+516$ & MK1500 & 164048.3 & 513632 & $32 \times 25$ & 0 & $8950 \pm 03$ & 122.9 & $148 \pm 06$ & $158 \pm 10$ & $1.29 \pm 0.22$ & $1.30 \pm 0.22$ & 9.67 & N93 \\
\hline $1707+565^{+}$ & SBS & 170703.3 & 563434 & $36 \times 21$ & 60 & $3323 \pm 19$ & 49.3 & $116 \pm 38$ & $169 \pm 60$ & $\mathbf{0 . 7 6} \pm \mathbf{0 . 2 0}$ & $0.77 \pm \mathbf{0 . 2 0}$ & 8.65 & N93 \\
\hline $1714+602$ & MK893 & 171423.0 & 601615 & $29 \times 21$ & 40 & $6014 \pm 11$ & 83.7 & $179 \pm 22$ & $200 \pm 35$ & $1.02 \pm 0.26$ & $1.03 \pm 0.26$ & 9.23 & N94* \\
\hline $1809+318$ & MK1121 & 180928.6 & 315055 & $50 \times 36$ & 0 & $4520 \pm 12$ & 63.7 & $84 \pm 23$ & $169 \pm 36$ & $2.68 \pm 0.23$ & $2.71 \pm \mathbf{0 . 2 3}$ & 9.41 & N94* \\
\hline $2116+020$ & MK513 & 211618.5 & 020302 & $39 \times 24$ & 90 & $5460 \pm 15$ & 73.0 & $190 \pm 28$ & $327 \pm 44$ & $2.97 \pm 0.19$ & $3.02 \pm 0.19$ & 9.58 & N93* \\
\hline $2127+021$ & MK900 & 212727.5 & 021139 & $169 \times 106$ & 43 & $1152 \pm 05$ & 17.4 & $106 \pm 08$ & $158 \pm 12$ & $2.00 \pm \mathbf{0 . 1 2}$ & $2.36 \pm 0.14$ & 8.23 & N93* \\
\hline $2220+377$ & MK908 & $\begin{array}{lll}22 & 20 & 28.2\end{array}$ & 374325 & $124 \times 64$ & -80 & $1240 \pm 02$ & 20.4 & $202 \pm 03$ & $218 \pm 05$ & $8.89 \pm \mathbf{0 . 3 8}$ & $10.19 \pm 0.43$ & 9.00 & N93* \\
\hline $2221+409$ & MK909 & $22 \quad 2117.5$ & 405541 & $46 \times 39$ & -30 & $4433 \pm 05$ & 62.0 & $168 \pm 09$ & $187 \pm 15$ & $2.22 \pm 0.32$ & $2.25 \pm 0.33$ & 9.31 & N93* \\
\hline $2246+315$ & MK921 & 224609.9 & 313100 & $39 \times 24$ & 33 & $3848 \pm 13$ & 53.6 & $137 \pm 27$ & $201 \pm 42$ & $2.30 \pm 0.27$ & $2.32 \pm 0.27$ & 9.20 & N93* \\
\hline $2311+129$ & MK528 & 231134.9 & 125419 & & & $2676 \pm 03$ & 37.1 & $189 \pm 06$ & $203 \pm 09$ & $8.26 \pm 0.82$ & & 9.43 & G92* \\
\hline $2329+286$ & MK930 & $\begin{array}{lll}23 & 29 & 29.5\end{array}$ & $\begin{array}{lll}28 & 40 & 18\end{array}$ & $26 \times 21^{\mathrm{e}}$ & 0 & $5485 \pm 10$ & 74.8 & $137 \pm 20$ & $190 \pm 31$ & $2.25 \pm 0.25$ & $2.26 \pm 0.25$ & 9.47 & N94 \\
\hline
\end{tabular}

$\mathbf{0 0 1 2 - 0 1 8}=\mathrm{UM} 219 \equiv \mathrm{KUG} 0012-018$

0034+356 - Wegner et al. (1993) found $F=1.67 \mathrm{Jy} \mathrm{km} \mathrm{s}^{-1}, W(50)=94 \mathrm{~km} \mathrm{~s}^{-1}$ and $W(20)=156 \mathrm{~km} \mathrm{~s}^{-1}$ in good agreement with our data.

$\mathbf{0 1 1 1 + 0 7 5}$ - Sc galaxy of the same brightness at $22^{\prime}$ to North. No velocity.

0125-065 - Our unpublished VLA data show a large HI envelope around the BCG. A HST study by Thuan et al. (1996) indicates an extremely compact and dense HII region in this galaxy. There is a galaxy at $10.5^{\mathrm{s}}$ to the West and $13^{\prime}$ to the South with no measured velocity.

$\mathbf{0 1 4 2 + 0 4 6} \equiv$ MCG $1-5-30$. Comet-like structure $(\sim 5 / \mathrm{h} \mathrm{kpc})$, with a very compact $\left(7^{\prime \prime}\right)$ HII region at the S edge.

$\mathbf{0 1 4 3 + 3 4 6} \equiv$ KUG $0143+346$. Wegner et al. (1993) obtained $F=3.26 \mathrm{Jy} \mathrm{km} \mathrm{s}^{-1}$ and $W_{20}=122 \mathrm{~km} \mathrm{~s}^{-1}$, about $30 \%$ higher than our flux measurement. In group: 3 Sc galaxies within inside $30^{\prime}$ with $V=5677,5653$ and $5577 \mathrm{~km} \mathrm{~s}^{-1}$

0144+024A - UM370 at $10.2^{\mathrm{s}}$ to the East and $4^{\prime}$ to the South is an O or B star according to Terlevich et al. (1991).

$\mathbf{0 2 1 4 + 2 9 2} \equiv \mathrm{V} \mathrm{Zw} 212$. Possible satellite galaxy from HI spectrum at $V=5365 \mathrm{~km} \mathrm{~s}^{-1}$ with $F=1.04 \mathrm{Jy} \mathrm{km} \mathrm{s}^{-1}, W_{50}=52 \mathrm{~km} \mathrm{~s}^{-1}, W_{20}=62 \mathrm{~km} \mathrm{~s}^{-1}$ (compact galaxy with $d(25)=5 \mathrm{kpc} 22^{\prime \prime}$ away). Keel \& van Soest (1992) found that this is a "probably paired" Markarian galaxy. The possible companion galaxy is at $2.9^{\prime}$, with no known velocity 
$\mathbf{0 2 2 5}-\mathbf{1 0 4} \equiv$ KUG 0225-104. In group with 0225-103.

0335-052 - With a metallicity of $Z_{\odot} / 41$, this is the most metal-deficient BCG known after I Zw $18\left(Z_{\odot} / 50\right)$. Detailed studies with the HST (Thuan et al. 1997; Thuan \& Izotov 1997), MMT (Izotov et al. 1997), Russian $6 \mathrm{~m}$ (Pustilnik et al. 1997) and Keck (Lipovetsky et al. 1999a) telescopes suggest that this galaxy is a young galaxy, containing stars not older than 100 Myr. A VLA map (Pustilnik et al. 1999) shows that the HI envelope is unusually large $\left(64 \times 24 \mathrm{kpc}^{2}\right)$ and seen nearly edge-on. As the HI gas structure is not well approximated by our adopted model of a gaussian disk, the beam correction is somewhat uncertain. However the single-dish and VLA HI masses agree quite well. NGC 1376 is at 37.3 to the West at $V=4155 \mathrm{~km} \mathrm{~s}^{-1}(\sim 100 / \mathrm{h} \mathrm{kpc} \mathrm{in} \mathrm{projection).} \mathrm{The} \mathrm{BCG} \mathrm{is} \mathrm{at} \mathrm{the} \mathrm{outskirts} \mathrm{of} \mathrm{a} \mathrm{medium} \mathrm{density} \mathrm{group} \mathrm{GH} 47$ (Garcia 1993$)$.

$\mathbf{0 7 4 1 + 5 3 5}$ - Galaxy at $7.2^{\mathrm{s}}$ to the East and $17^{\prime}$ to the North. In the same group with $2 \mathrm{SB}$ galaxies with velocities of $5641 \mathrm{~km} \mathrm{~s}^{-1}$ and $5679 \mathrm{~km} \mathrm{~s}{ }^{-1}$, inside a radius of $30^{\prime}$. $\mathbf{0 7 4 3}+\mathbf{5 5 0} \equiv \mathrm{MCG} 9-13-55 \equiv \mathrm{VV} 654$, pair? In group? Sb galaxy NGC 2446 at $\sim 25^{\prime}$ with $\Delta V=180 \mathrm{~km} \mathrm{~s}^{-1}$

$\mathbf{0 7 4 9}+\mathbf{5 6 8}$ - Possible HI satellite at $V=5339 \mathrm{~km} \mathrm{~s}^{-1}, F=0.24 \pm 0.11 \mathrm{Jy} \mathrm{km} \mathrm{s}^{-1}, W_{50}=70 \mathrm{~km} \mathrm{~s}^{-1}$. This HI component may be associated with a brighter galaxy at $\sim 2^{\prime}$ to the West and $\sim 1.7^{\prime}$ to the North, or with a fainter galaxy at $0.2^{\prime}$ to the East and $\sim 6^{\prime}$ to the South, or with a very faint fuzz at $10^{\prime \prime}$ to the East and $30^{\prime \prime}$ to the South.

$\mathbf{0 8 4 7}+\mathbf{6 1 2} \equiv$ MCG 10-13-25. Compact galaxy with a bright nucleus and two tails.

$\mathbf{0 9 1 7}+\mathbf{5 2 7} \equiv \mathrm{KUG} 0917+527$.

$\mathbf{0 9 3 0}+\mathbf{5 5 4} \equiv \mathrm{I}$ Zw $18 \equiv$ UGCA 166 . The most metal-deficient BCG known (1/50 of solar metallicity) and a young galaxy (stars not older than $100 \mathrm{Myr}$, Izotov \& Thuan 1998$)$. $\mathbf{0 9 3 9 + 5 9 2} \equiv$ UGC 5179. Spiral galaxy seen almost face-on. The bright SO galaxy NGC 2950 is 3.5 mag brighter at $25.3^{\mathrm{s}}$ to the West and $7^{\prime}$ to the South, $V=1378 \mathrm{~km} \mathrm{~s}^{-1}$ $(\sim 30 / \mathrm{h} \mathrm{kpc} \mathrm{in} \mathrm{projection).}$

$\mathbf{0 9 4 0}+\mathbf{6 6 2} \equiv$ NGC $2909 \equiv$ UGC 5188. Haynes et al. (1988) obtained $F=2.02 \mathrm{Jy} \mathrm{km} \mathrm{s}{ }^{-1}$ and $W_{50}=130 \mathrm{~km} \mathrm{~s}^{-1}, W_{20}=188 \mathrm{~km} \mathrm{~s}{ }^{-1}$ consistent with our data.

$\mathbf{0 9 4 0}+\mathbf{5 4 4} \equiv$ KUG $0940+544$. KUG $0940+543,1$ mag brighter galaxy at $10^{\mathrm{s}}$ to the West and $1.5^{\prime}$ to the South. Its velocity is about $27000 \mathrm{~km} \mathrm{~s}^{-1}(6 \mathrm{~m} \mathrm{data)}$

$\mathbf{0 9 4 2}+\mathbf{5 7 3} \equiv \mathrm{KUG} 0942+573$.

$\mathbf{0 9 4 6}+\mathbf{4 8 7} \equiv \mathrm{KUG} 0946+487$

$\mathbf{0 9 4 6}+\mathbf{5 5 8} \equiv$ UGCA $184 \equiv$ KUG 0946+588, spiral, Thuan \& Martin (1981) obtained $F=1.23 \pm 0.8 \mathrm{Jy} \mathrm{km} \mathrm{s}^{-1}, W_{50}=69 \mathrm{~km} \mathrm{~s}^{-1}, W_{20}=104 \mathrm{~km} \mathrm{~s}^{-1}$.

$\mathbf{1 0 0 1 + 5 5 5} \equiv$ UGC $5421 \equiv$ MCG 9-17-18, Dwarf $(\sim 9 \mathrm{kpc})$ galaxy seen edge-on. Schneider et al. (1992) obtained $\left.F=4.19 \pm 0.6 \mathrm{Jy} \mathrm{km} \mathrm{s}^{-1}, W_{50}=100 \mathrm{~km} \mathrm{~s}^{-1}, W_{20}=116 \mathrm{~km} \mathrm{~s}^{-1}\right)$, Haynes \& Giovanelli (1991) obtained $F=4.53 \mathrm{Jy} \mathrm{km} \mathrm{s}^{-1}, W_{50}=105 \mathrm{~km} \mathrm{~s}^{-1}, W_{20}=127 \mathrm{~km} \mathrm{~s}^{-1}$ and Theureau et al. (1998) obtained $F=4.4 \pm 0.5 \mathrm{Jy} \mathrm{km} \mathrm{s}{ }^{-1}, W_{50}=110 \mathrm{~km} \mathrm{~s}{ }^{-1}$, $W_{20}=119 \mathrm{~km} \mathrm{~s}^{-1}$ in good agreement with our data.

$\mathbf{1 0 0 6}+\mathbf{5 7 8} \equiv$ KUG $1006+578$.

$\mathbf{1 1 1 4}+\mathbf{5 8 7}$ - In group? Two galaxies inside a $30^{\prime}$ radius with velocities of $1772 \mathrm{~km} \mathrm{~s}^{-1}$ and $1808 \mathrm{~km} \mathrm{~s}^{-1}$

$\mathbf{1 1 1 9}+\mathbf{5 8 6}$ - Galaxy $2 \mathrm{mag}$ brighter, $11.4^{\mathrm{s}}$ to the West and $18^{\prime}$ to the South, with no known velocity. Background HI emission at $V=1943 \mathrm{~km} \mathrm{~s}^{-1} \mathrm{with} F=0.37 \pm 0.18 \mathrm{Jy} \mathrm{km} \mathrm{s}{ }^{-1}$ $W_{50}=36 \mathrm{~km} \mathrm{~s}^{-1}$. It may come from either a pair of galaxies at $\sim 1^{\prime}$ to the East and $\sim 4^{\prime}$ to the South, or a faint galaxy at $8^{\prime \prime}$ to the East and $\sim 7.3^{\prime}$ to the North.

$\mathbf{1 1 2 3}+\mathbf{6 4 4} \equiv \mathrm{UGC} 6448 \equiv$ MCG 11-14-25. Dwarf Irr disk with 2 bright knots. Thuan \& Martin (1981) obtained $F=4.2 \pm 1.06 \mathrm{Jy} \mathrm{km} \mathrm{s}^{-1}, W_{50}=60 \mathrm{~km} \mathrm{~s}{ }^{-1}, W_{20}=87 \mathrm{~km} \mathrm{~s}^{-1}$ In group? UGC 6390 inside of $30^{\prime}$ radius at $V=1009 \mathrm{~km} \mathrm{~s}^{-1}(\sim 75 / \mathrm{h} \mathrm{kpc} \mathrm{in} \mathrm{projection).}$

$\mathbf{1 2 1 1}+\mathbf{5 4 0}$ - One of the smallest objects in our sample.

$\mathbf{1 2 1 3 + 5 9 7} \equiv$ MCG 10-18-13. Spiral seen almost face-on. In group? NGC 4195 inside $30^{\prime}$ radius at $V=4350 \mathrm{~km} \mathrm{~s}^{-1}$

$\mathbf{1 3 1 7 + 5 2 3 A} \equiv$ MCG 9-22-47. Observation was made at $13^{\mathrm{s}}$ to the West. A correction factor of 1.27 has been taken into account for the integrated flux density. With this offset, the integrated flux density contribution of the nearby galaxy SBS $1317+523 \mathrm{~B}$ ( $\equiv \mathrm{MK} 251$, at $20^{\mathrm{s}}$ to the East and $1.5^{\prime}$ to the North, at $V=4618 \mathrm{~km} \mathrm{~s}{ }^{-1}$ ) is less than $15 \%$ of the measured integrated flux density of SBS 1317+523A, which is, after subtraction, of $4.2 \mathrm{Jy} \mathrm{km} \mathrm{s}^{-1}$. In group with the companion galaxy MCG 9-22-48, located at $3^{\mathrm{s}}$ to the East and $30^{\prime \prime}$ to the South, with $V=4444 \pm 40 \mathrm{~km} \mathrm{~s}^{-1}$, yielding no confusion, with SBS $1317+523 \mathrm{~B}$, and with SBS $1318+520$ at $25^{\mathrm{s}}$ to the East, $18^{\prime}$ to the South and $V=$ $4740 \mathrm{~km} \mathrm{~s}^{-1}$

1318+520 $\equiv$ MCG 9-22-51. In group with SBS 1317+523A and B and MCG 9-22-48 (see the note for SBS 1317+523A).

$1323+483$ - Foreground HI source at $V=4602 \mathrm{~km} \mathrm{~s}^{-1}$ with $F=0.15 \pm 0.07 \mathrm{Jy} \mathrm{km} \mathrm{s}{ }^{-1}, W_{50}=32 \mathrm{~km} \mathrm{~s}^{-1}$. Fainter galaxy at $3^{\prime \prime}$ to the West and $24^{\prime \prime}$ to the South.

$\mathbf{1 3 3 2}+\mathbf{5 1 8} \equiv \mathrm{NGC} 5238 \equiv \mathrm{UGC} 8565 \equiv \mathrm{I} \mathrm{Zw} 64 \equiv \mathrm{VV} 828 \equiv \mathrm{CP} 384 \mathrm{ab}$

$\mathbf{1 3 5 8}+\mathbf{5 5 4 E}$ - Edge-on spiral NGC $5422\left(B=12^{\mathrm{m}} 8\right)$ about $6^{\prime}$ to the SE with $V=1785 \mathrm{~km} \mathrm{~s}^{-1}$

$\mathbf{1 4 2 2}+\mathbf{5 7 3} \equiv$ KUG $1422+573$. Spheroid with 2 tails along major axis.

$\mathbf{1 4 3 0}+\mathbf{5 9 6}$ - Edge-on Spiral. There is also a HI component at $V=1955 \mathrm{~km} \mathrm{~s}^{-1}$ with $F=0.43 \pm 0.17 \mathrm{Jy} \mathrm{km} \mathrm{s}^{-1}, W_{50}=46 \mathrm{~km} \mathrm{~s}^{-1}$. This may be associated with a galaxy 2 mag fainter at $4^{\prime}$ to the North. In group. Two SBc galaxies inside $30^{\prime}$ radius at velocities of $2001 \mathrm{~km} \mathrm{~s}^{-1}$ and $1918 \mathrm{~km} \mathrm{~s}^{-1}$.

$\mathbf{1 4 3 0}+\mathbf{5 2 6} \mathbf{A} \equiv$ KUG $1430+526$

$\mathbf{1 5 0 4 + 5 1 4 N} \equiv$ KUG $1504+514 \equiv$ CGCG $274-13$. Sc galaxy UGC 9702 at $8^{\prime}$ to the SW with unknown velocity.

$\mathbf{1 5 0 6}+\mathbf{5 5 3} \equiv$ Sc galaxy UGC $9737 \equiv$ MCG 9-25-23. In group. 2 Sc galaxies inside $30^{\prime}$ radius at velocities $3128 \mathrm{~km} \mathrm{~s}^{-1}$ and $3421 \mathrm{~km} \mathrm{~s}^{-1}$

$\mathbf{1 5 0 9 + 5 2 7} \equiv$ KUG 1509+527. Paired with Sdm galaxy MCG 9-25-31, 0.4 mag brighter and with $a(25)=1^{\prime}$, at $36^{\prime \prime}$ to the West and $6^{\prime}$ to the South, at $V=3314 \mathrm{~km} \mathrm{~s}^{-1}$

$\left(F=0.34 \pm 0.14 \mathrm{Jy} \mathrm{km} \mathrm{s}^{-1}, W_{50}=86 \mathrm{~km} \mathrm{~s}^{-1}\right)$. In group. 3 galaxies with velocities equal to $3526 \mathrm{~km} \mathrm{~s}^{-1}, 3360 \mathrm{~km} \mathrm{~s}^{-1}$ and $3470 \mathrm{~km} \mathrm{~s}^{-1}$ inside a $30^{\prime}$ radius. 
Notes to Table 1 (continued):

$\mathbf{1 5 1 9 + 4 9 6}$ - Two galaxies at $3.7^{\mathrm{s}}$ to the West and $23^{\prime}$ to the South, with unknown velocities.

$1551+601 \mathrm{~A}$ - The HI and optical velocities differ by $300 \mathrm{~km} \mathrm{~s}^{-1}$. Possible faint foreground HI emission at $V=2516 \mathrm{~km} \mathrm{~s}^{-1}, F=0.16 \pm 0.11 \mathrm{Jy} \mathrm{km} \mathrm{s}{ }^{-1}, W_{50}=28 \mathrm{~km} \mathrm{~s}{ }^{-1}$

which could be associated either to a faint galaxy at $3.3^{\prime}$ to the South, or to a faint galaxy with bright knots $76^{\prime \prime}$ to the East and $\sim 6^{\prime}$ to the South.

$1714+602$ - There is a second component of the HI profile with $V=6070 \mathrm{~km} \mathrm{~s}^{-1}$ and $F=0.36 \pm 0.12 \mathrm{Jy} \mathrm{km} \mathrm{s}{ }^{-1}$. It may correspond to faint galaxy at $\sim 1.5^{\prime}$ to South.

$\mathbf{1 8 0 9}+\mathbf{3 1 8}$ Keel \& van Soest (1992) found that this is a "probably paired" Markarian galaxy. The possible companion galaxy is at $0.3^{\prime}$, with no known velocity and 2.2 mag fainter than SBS $1809+318$

2116+020 Keel \& van Soest (1992) found that this is a "probably paired" Markarian galaxy. The possible companion galaxy is at $2.2^{\prime}$, with no known velocity and 1.8 mag fainter than SBS $2116+020$

$\mathbf{2 1 2 7}+\mathbf{0 2 1} \equiv \mathrm{NGC} 7077 \equiv$ Arakelian 549. Lewis (1987) obtained $F=1.97 \mathrm{Jy} \mathrm{km} \mathrm{s}^{-1}, W_{50}=121 \mathrm{~km} \mathrm{~s}^{-1}, W_{20}=131 \mathrm{~km} \mathrm{~s}^{-1}$, in good agreement with our data

$\mathbf{2 2 2 0}+\mathbf{3 7 7} \equiv \mathrm{UGC} 12009$. Wegner et al. (1993) obtained $F=8.78 \mathrm{Jy} \mathrm{km} \mathrm{s}^{-1}, W_{50}=202 \mathrm{~km} \mathrm{~s}^{-1}$ and $W_{20}=189 \mathrm{~km} \mathrm{~s}^{-1}$ in good agreement with our data.

2221+409 - Sm galaxy UGC 12027 at $\sim 10^{\prime}$ with $V=4130 \mathrm{~km} \mathrm{~s}^{-1}$ (Schneider et al. 1992). In group?

2246+315 - Jackson et al. (1987) obtained $F=3.37 \mathrm{Jy} \mathrm{km} \mathrm{s}^{-1}, W_{20}=206 \mathrm{~km} \mathrm{~s}^{-1}, 60 \%$ higher than our flux.

$\mathbf{2 3 1 1 + 1 2 9} \equiv$ UGC 12434 


\begin{tabular}{|c|c|c|c|c|c|c|c|c|c|c|c|c|c|}
\hline \multirow[t]{2}{*}{ IAU name } & \multirow{2}{*}{$\begin{array}{c}\text { Other } \\
\text { name or } \\
\text { prefix }\end{array}$} & \multicolumn{2}{|c|}{ Coord. (1950.0) } & \multirow{2}{*}{$\begin{array}{l}\text { Angul. } \\
\text { size } \\
\text { axb }\end{array}$} & \multirow[t]{2}{*}{ PA } & \multirow{2}{*}{$\begin{array}{c}V(\mathbf{H I}) \\
\mathrm{km} \mathrm{s}^{-1}\end{array}$} & \multirow{2}{*}{$\begin{array}{l}\text { Dist. } \\
\text { Mpc } \\
\text { Vir }\end{array}$} & \multirow{2}{*}{$\begin{array}{l}W_{50} \\
\mathbf{k m ~ s}^{-1}\end{array}$} & \multirow{2}{*}{$\begin{array}{l}W_{20} \\
\mathrm{~km} \mathrm{~s}^{-1}\end{array}$} & \multirow{2}{*}{$\begin{array}{l}\text { Obs. Flux } \\
\text { Jy km s s }\end{array}$} & \multirow{2}{*}{$\begin{array}{l}\text { Cor. Flux } \\
\text { Jy km s s}\end{array}$} & \multirow{2}{*}{$\begin{array}{c}\text { Log } \\
M(\mathbf{H I}) / \\
M_{\odot}\end{array}$} & \multirow{2}{*}{$\begin{array}{c}R \\
\& \\
N\end{array}$} \\
\hline & & $\begin{array}{l}\text { R.A. } \\
\text { h m s }\end{array}$ & $\begin{array}{l}\text { Dec. } \\
0, \prime \prime\end{array}$ & & & & & & & & & & \\
\hline 1 & 2 & 3 & 4 & 5 & 6 & 7 & 8 & 9 & 10 & 11 & 12 & 13 & 14 \\
\hline $0028+081$ & MK552 & $\begin{array}{lll}00 & 28 & 43.9\end{array}$ & 081159 & $103 \times 61^{\mathrm{e}}$ & $\mathbf{0}$ & $4368 \pm 11$ & 58.5 & $239 \pm 21$ & $337 \pm \mathbf{3 3}$ & $23.60 \pm 1.36$ & & 10.28 & G92* \\
\hline $0127+307 \mathrm{~A}$ & KUG & 012753.6 & 304329 & $33 \times 20^{\mathrm{e}}$ & -30 & $4958 \pm 04$ & 67.5 & $67 \pm 08$ & $85 \pm 12$ & $1.46 \pm 0.26$ & $1.47 \pm 0.26$ & 9.20 & N94* \\
\hline $0136+328$ & KUG & 013620.8 & 325152 & $27 \times 22^{\mathrm{e}}$ & $\mathbf{0}$ & $6230 \pm 21$ & 84.4 & $34 \pm 42$ & $112 \pm 66$ & $0.63 \pm 0.23$ & $0.63 \pm \mathbf{0 . 2 3}$ & 9.03 & N93* \\
\hline $0201+284^{*}$ & MK365 & 020126.0 & 282457 & $70 \times 55^{\mathrm{e}}$ & 90 & $5151 \pm 05$ & 69.8 & $63 \pm 11$ & $74 \pm 17$ & $0.25 \pm 0.13$ & $0.26 \pm 0.13$ & 8.48 & N94* \\
\hline $0207-080$ & MK1023 & 020720.6 & -080105 & $26 \times 20^{\mathrm{e}}$ & 0 & $3793 \pm 12$ & 49.5 & $100 \pm 24$ & $139 \pm 38$ & $1.13 \pm 0.24$ & $1.13 \pm 0.24$ & 8.82 & N94* \\
\hline $0310-054$ & MK604 & $\begin{array}{llll}03 & 10 & 18.4\end{array}$ & -052719 & $48 \times 23^{\mathrm{e}}$ & 10 & $2177 \pm 05$ & 28.6 & $144 \pm 10$ & $186 \pm 15$ & $4.63 \pm 0.38$ & $4.66 \pm \mathbf{0 . 3 8}$ & 8.95 & N93* \\
\hline 0335-057A & SBS & 033544.6 & -054211 & $25 \times 25^{\mathrm{e}}$ & 0 & $6617 \pm 07$ & 86.6 & $201 \pm 13$ & $222 \pm 21$ & $2.70 \pm 0.45$ & $2.72 \pm 0.45$ & 9.68 & N93* \\
\hline $0743+591 B$ & SBS & 074332.2 & 590752 & $64 \times 31$ & 45 & $6340 \pm 14$ & 87.5 & $88 \pm 28$ & $130 \pm 44$ & $0.84 \pm \mathbf{0 . 2 9}$ & $\mathbf{0 . 8 6} \pm \mathbf{0 . 3 0}$ & 9.19 & N93* \\
\hline $0750+603 \mathrm{AB}$ & SBS & 075053.7 & 601910 & $30 \times 18^{\mathrm{e}}$ & 65 & $10844 \pm 17$ & 146.9 & $183 \pm 34$ & $238 \pm 54$ & $1.41 \pm 0.27$ & $1.42 \pm 0.27$ & 9.86 & N93* \\
\hline $0755+587$ & SBS & 075542.8 & 584512 & $83 \times 66^{\mathrm{e}}$ & 10 & $5720 \pm 12$ & 79.4 & $210 \pm 24$ & $253 \pm 38$ & $5.55 \pm 0.92$ & & 9.92 & G92* \\
\hline $0756+611^{*}$ & SBS & $\begin{array}{l}075629.3 \\
\end{array}$ & 610725 & $20 \times 16^{\mathrm{e}}$ & -20 & $6269 \pm 05$ & 86.7 & $93 \pm 09$ & $100 \pm 15$ & $0.70 \pm 0.41$ & & 9.09 & G92* \\
\hline 0814+579A & SBS & $\begin{array}{lll}08 & 1408.9\end{array}$ & 575451 & $53 \times 32$ & -45 & $8048 \pm 23$ & 110.0 & $142 \pm 46$ & $253 \pm 72$ & $2.87 \pm 0.41$ & $2.93 \pm 0.42$ & 9.92 & N93* \\
\hline $0844+703$ & MK95 & 084433.4 & 702053 & $49 \times 24$ & -33 & $3649 \pm 07$ & 52.9 & $286 \pm 13$ & $294 \pm 20$ & $4.01 \pm 2.06$ & & 9.42 & G92* \\
\hline $0912+599^{+*}$ & MK19 & $\begin{array}{lll}09 & 1253.5\end{array}$ & 595853 & $65 \times 30$ & 60 & $4150 \pm 13$ & 59.2 & $144 \pm 26$ & $206 \pm 40$ & $2.33 \pm \mathbf{0 . 3 7}$ & $2.41 \pm \mathbf{0 . 3 8}$ & 9.30 & N93* \\
\hline $0926+606 \mathrm{AB}^{+}$ & SBS & $\begin{array}{ll}09 & 2620.1\end{array}$ & 604002 & $43 \times 26$ & 90 & $4050 \pm 10$ & 59.1 & $148 \pm 20$ & $175 \pm 32$ & $2.53 \pm 0.53$ & $2.59 \pm 0.54$ & 9.33 & G93* \\
\hline $1011+601^{+}$ & SBS & 101131.5 & 600607 & $16 \times 13^{\mathrm{e}}$ & 90 & $2160 \pm 17$ & 33.7 & $42 \pm 34$ & $\mathbf{9 5} \pm \mathbf{5 3}$ & $0.52 \pm 0.19$ & $0.52 \pm 0.19$ & 8.14 & N93* \\
\hline $1028+566^{*}$ & SBS & 102855.3 & 563706 & $35 \times 28$ & -50 & $7261 \pm 26$ & 100.0 & $148 \pm 52$ & $190 \pm 82$ & $0.56 \pm 0.20$ & $0.57 \pm 0.20$ & 9.13 & N93* \\
\hline $1054+504^{+*}$ & SBS & 105402.7 & 502428 & $42 \times 27$ & -45 & $1375 \pm 31$ & 23.4 & $87 \pm 62$ & $158 \pm \mathbf{9 7}$ & $0.58 \pm 0.21$ & $0.59 \pm 0.21$ & 7.88 & N93* \\
\hline $1057+511 A^{*}$ & U6074 & 105702.7 & 511014 & $78 \times 52$ & 90 & $2885 \pm 14$ & 43.0 & $84 \pm 28$ & $152 \pm 43$ & $5.25 \pm 0.85$ & & 9.36 & G92* \\
\hline $1114+517^{+}$ & MK1445 & 111442.7 & 514216 & $41 \times 29$ & 0 & $2838 \pm 30$ & 43.1 & $\mathbf{3 0} \pm \mathbf{1 0}$ & $50 \pm \mathbf{2 0}$ & $0.90 \pm 0.26$ & $0.90 \pm \mathbf{0 . 2 6}$ & 8.56 & N94* \\
\hline $1114+5145$ & U6309 & 111457.0 & 514517 & $105 \times 83$ & -60 & $2870 \pm 06$ & 43.4 & $223 \pm 13$ & $257 \pm 20$ & $4.12 \pm 0.35$ & $4.53 \pm 0.39$ & 9.26 & N96* \\
\hline $1123+576^{+*}$ & SBS & 112323.2 & 573742 & $24 \times 22$ & $\mathbf{0}$ & $289 \pm 07$ & 6.3 & $32 \pm 14$ & $42 \pm 23$ & $0.12 \pm 0.08$ & $0.12 \pm 0.08$ & 6.05 & N94* \\
\hline $1125+562^{+}$ & SBS & 112516.8 & 561303 & $33 \times 25$ & -40 & $5602 \pm 11$ & 78.4 & $74 \pm 22$ & $95 \pm 34$ & $0.47 \pm 0.23$ & $0.47 \pm \mathbf{0 . 2 3}$ & 8.83 & N95* \\
\hline $1125+524$ & SBS & 112550.8 & 522721 & $44 \times 22$ & 55 & $3075 \pm 32$ & 45.6 & $53 \pm 65$ & $148 \pm 101$ & $0.71 \pm 0.19$ & $0.72 \pm 0.20$ & 8.55 & N94* \\
\hline $1129+576^{+}$ & SBS & 112915.5 & 573917 & $65 \times 18$ & -15 & $1566 \pm 02$ & 26.3 & $89 \pm 04$ & $119 \pm 05$ & $21.62 \pm 0.80$ & $21.74 \pm \mathbf{0 . 8 5}$ & 9.55 & G92* \\
\hline $1136+607^{+}$ & SBS & 113626.3 & 604722 & $29 \times 21$ & 45 & $3481 \pm 08$ & 51.0 & $278 \pm 17$ & $305 \pm 26$ & $4.18 \pm 0.57$ & $4.21 \pm 0.58$ & 9.41 & N93* \\
\hline $1137+589^{+*}$ & SBS & 113748.4 & $\begin{array}{lll}58 & 5510\end{array}$ & $20 \times 14$ & 0 & $2032 \pm 09$ & 32.5 & $110 \pm 17$ & $124 \pm 27$ & $0.85 \pm 0.39$ & $\mathbf{0 . 8 5} \pm \mathbf{0 . 3 9}$ & 8.33 & N94* \\
\hline $1144+591^{*}$ & SBS & 114421.9 & 590950 & $28 \times 22$ & 20 & $2488 \pm 11$ & 38.1 & $84 \pm 23$ & $148 \pm 36$ & $2.75 \pm 0.42$ & $2.78 \pm 0.42$ & 8.97 & N93* \\
\hline $1147+520^{+}$ & SBS & 114716.4 & 520051 & $28 \times 23$ & 0 & $1257 \pm 07$ & 22.2 & $148 \pm 15$ & $169 \pm 23$ & $1.79 \pm 0.39$ & $1.80 \pm \mathbf{0 . 3 9}$ & 8.32 & N93* \\
\hline $1149+596^{+*}$ & SBS & 114955.9 & $\begin{array}{lll}59 & 3937\end{array}$ & $30 \times 22^{\mathrm{e}}$ & 40 & $3351 \pm 25$ & 49.0 & $42 \pm 51$ & $116 \pm 80$ & $0.55 \pm 0.21$ & $0.55 \pm 0.21$ & 8.49 & N94* \\
\hline $1159+516 \mathrm{~B}^{+}$ & SBS & 115931.8 & $\begin{array}{llll}51 & 40 & 18\end{array}$ & $22 \times 22^{\mathrm{e}}$ & 0 & $4424 \pm 08$ & 8.3 & $35 \pm 16$ & $48 \pm 24$ & $0.37 \pm 0.25$ & $0.37 \pm 0.25$ & 6.78 & N94* \\
\hline $1216+460$ & MK1471 & 121610.8 & 460345 & $30 \times 17$ & -28 & $5796 \pm 06$ & 80.8 & $232 \pm 11$ & $244 \pm 17$ & $4.65 \pm 0.90$ & & 9.86 & G92* \\
\hline $1216+551^{*}$ & SBS & 121655.5 & $\begin{array}{lll}55 & 08 & 13\end{array}$ & $20 \times 20^{\mathrm{e}}$ & 0 & $5195 \pm 06$ & 73.2 & $32 \pm 11$ & $42 \pm 18$ & $0.47 \pm 0.32$ & $0.47 \pm \mathbf{0 . 3 2}$ & 8.77 & N94* \\
\hline $1221+585^{+}$ & SBS & 122119.8 & 583123 & $38 \times 25$ & 70 & $4610 \pm 09$ & 65.6 & $88 \pm 17$ & $115 \pm 26$ & $0.75 \pm \mathbf{0 . 1 8}$ & $\mathbf{0 . 7 6} \pm \mathbf{0 . 1 8}$ & 8.89 & N94* \\
\hline $1221+545 \mathrm{~B}^{+*}$ & SBS & 122158.6 & 543124 & $15 \times 11$ & -50 & $5704 \pm 57$ & 78.1 & $40 \pm 129$ & $129 \pm 178$ & $0.16 \pm 0.16$ & $0.16 \pm 0.16$ & 8.36 & N94* \\
\hline $1222+614^{+}$ & SBS & 122244.5 & 612545 & $38 \times 34$ & 75 & $706 \pm 02$ & 14.1 & $67 \pm 04$ & $84 \pm 07$ & $5.26 \pm 0.55$ & $5.34 \pm \mathbf{0 . 5 6}$ & 8.40 & N93* \\
\hline $1240+554 \mathrm{C}$ & SBS & 124036.6 & 552509 & $139 \times 39$ & -45 & $4797 \pm 19$ & 68.1 & $74 \pm 38$ & $159 \pm 59$ & $1.45 \pm 0.27$ & $1.59 \pm \mathbf{0 . 3 0}$ & 9.24 & N94* \\
\hline $1242+549^{+}$ & SBS & 124220.2 & 545951 & $41 \times 33$ & 45 & $4757 \pm 12$ & 67.2 & $63 \pm 23$ & $106 \pm 37$ & $1.33 \pm 0.21$ & $1.35 \pm 0.21$ & 9.16 & N93* \\
\hline $1314+605^{+*}$ & SBS & 131454.1 & 603420 & $45 \times 23$ & -70 & $1848 \pm 07$ & 30.3 & $42 \pm 13$ & $53 \pm 21$ & $0.52 \pm \mathbf{0 . 3 2}$ & $0.53 \pm \mathbf{0 . 3 2}$ & 8.06 & N96* \\
\hline $1317+523 \mathrm{~B}^{+}$ & MK251 & 131756.3 & 521847 & $35 \times 26$ & 90 & $4618 \pm 16$ & 65.8 & $131 \pm 31$ & $228 \pm 49$ & $3.85 \pm 0.36$ & $3.90 \pm \mathbf{0 . 3 6}$ & 9.60 & N94* \\
\hline $1319+579 \mathrm{AB}^{+}$ & SBS & 131928.4 & 575736 & $83 \times 32$ & 39 & $2097 \pm 10$ & 33.5 & $136 \pm 20$ & $218 \pm 31$ & $10.25 \pm 0.85$ & & 9.43 & G91* \\
\hline $1340+529^{+*}$ & MK1480 & 134056.0 & 525735 & $35 \times 24$ & -15 & $1801 \pm 06$ & 29.6 & $42 \pm 00$ & $84 \pm 00$ & $1.95 \pm 0.32$ & $1.96 \pm 0.32$ & 8.61 & N94* \\
\hline $1341+529^{+*}$ & MK1481 & 134103.6 & 525622 & $48 \times 36$ & 60 & $1797 \pm 09$ & 29.7 & $55 \pm 12$ & $96 \pm 19$ & $3.16 \pm 0.43$ & & 8.82 & G91* \\
\hline $1401+490^{+}$ & CG337 & 140150.0 & 490018 & $42 \times 25$ & 45 & $777 \pm 17$ & 14.9 & $48 \pm 33$ & $102 \pm 52$ & $0.83 \pm 0.28$ & $0.84 \pm \mathbf{0 . 2 8}$ & 7.64 & N93* \\
\hline $1450+439$ & CG564 & 145009.8 & $43 \quad 5527$ & $100 \times 77$ & -10 & $2480 \pm 04$ & 38.2 & $136 \pm 08$ & $160 \pm 13$ & $7.32 \pm 0.69$ & $7.76 \pm \mathbf{0 . 7 3}$ & 9.43 & N94* \\
\hline $1523+519^{+*}$ & CG707 & 152351.6 & 515522 & $36 \times 18$ & 52 & $3645 \pm 61$ & 53.1 & $42 \pm 123$ & $196 \pm 192$ & $0.39 \pm 0.26$ & $0.39 \pm \mathbf{0 . 2 6}$ & 8.41 & N94* \\
\hline
\end{tabular}




\begin{tabular}{|c|c|c|c|c|c|c|c|c|c|c|c|c|c|}
\hline \multirow[t]{2}{*}{ IAU name } & \multirow{2}{*}{$\begin{array}{c}\text { Other } \\
\text { name or } \\
\text { prefix }\end{array}$} & \multicolumn{2}{|c|}{ Coord. (1950.0) } & \multirow{2}{*}{$\begin{array}{c}\text { Angul. } \\
\text { size } \\
\text { axb }\end{array}$} & \multirow[t]{2}{*}{$\overline{\text { PA }}$} & \multirow{2}{*}{$\begin{array}{l}V(\mathbf{H I}) \\
\mathrm{km} \mathrm{s}^{-1}\end{array}$} & \multirow{2}{*}{$\begin{array}{l}\text { Dist. } \\
\text { Mpc } \\
\text { Vir }\end{array}$} & \multirow{2}{*}{$\begin{array}{c}W_{50} \\
\mathbf{k m ~ s}^{-1}\end{array}$} & \multirow{2}{*}{$\begin{array}{c}W_{20} \\
\mathbf{k m ~ s}^{-1}\end{array}$} & \multirow{2}{*}{$\begin{array}{c}\text { Obs. Flux } \\
\text { Jy km s }^{-1}\end{array}$} & \multirow{2}{*}{$\begin{array}{c}\text { Cor. Flux } \\
\text { Jy km s }^{-1}\end{array}$} & \multirow{2}{*}{$\begin{array}{c}\mathbf{L o g} \\
M(\mathbf{H I}) / \\
M_{\odot}\end{array}$} & \multirow{2}{*}{$\begin{array}{c}R \\
\& \\
N\end{array}$} \\
\hline & & $\begin{array}{c}\text { R.A. } \\
\text { h m s }\end{array}$ & $\begin{array}{l}\text { Dec. } \\
\circ,{ }^{\prime}, \prime\end{array}$ & & & & & & & & & & \\
\hline 1 & 2 & 3 & 4 & 5 & 6 & 7 & 8 & 9 & 10 & 11 & 12 & 13 & 14 \\
\hline $1533+574^{+}$ & SBS & 153303.3 & 572704 & $40 \times$ & -60 & 310 & 49.0 & $152=$ & $2=$ & $4.89 \pm$ & $4.96 \pm$ & 9.45 & N93* $^{*}$ \\
\hline $1533+469^{*}$ & IZw116 & $\begin{array}{ll}15 & 33 \\
23.9\end{array}$ & 465848 & $35 \times 19$ & -50 & $5687 \pm 15$ & 79.8 & $91 \pm 30$ & $153 \pm 48$ & $1.43 \pm 0.28$ & $1.44 \pm 0.28$ & 9.34 & N93* $^{*}$ \\
\hline $1542+573 B^{*}$ & SBS & 154241.1 & 572319 & $82 \times 22$ & -45 & $4027 \pm 08$ & 58.3 & $283 \pm 16$ & $301 \pm 25$ & $13.13 \pm 2.36$ & $13.52 \pm 2.39$ & 10.02 & N94* \\
\hline $1555+515^{+}$ & SBS & 155540.2 & 513054 & $17 \times 14$ & 38 & $4004 \pm 20$ & 55.3 & $137 \pm 40$ & $196 \pm 63$ & $1.20 \pm 0.25$ & $1.20 \pm 0.25$ & 8.94 & N94* \\
\hline $1558+585$ & SBS & 155854.8 & 583111 & $13 \times 11$ & 90 & $4218 \pm 13$ & 60.9 & $158 \pm 27$ & $211 \pm 42$ & $1.40 \pm 0.25$ & $1.40 \pm 0.25$ & 9.09 & N95* \\
\hline $1632+578^{+*}$ & SBS & 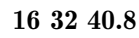 & 575238 & $24 \times 12$ & -42 & $5530 \pm 05$ & 69.7 & $42 \pm 11$ & $53 \pm 17$ & $0.20 \pm 0.11$ & $0.20 \pm 0.11$ & 8.36 & N94* \\
\hline $2304+097^{*}$ & MK1129 & 230409.7 & 094402 & $25 \times 25^{\mathrm{e}}$ & 0 & $4794 \pm 13$ & 64.6 & $\mathbf{6 3} \pm \mathbf{2 6}$ & $87 \pm 40$ & $0.45 \pm 0.20$ & $0.45 \pm 0.20$ & 8.65 & $\mathrm{~N}^{2}{ }^{*}$ \\
\hline $2311+235^{*}$ & MK317 & $\begin{array}{lll}23 & 11 & 20.7\end{array}$ & 233254 & $90 \times 60$ & -20 & $6202 \pm 05$ & 84.0 & $53 \pm 10$ & $63 \pm 15$ & $0.16 \pm 0.10$ & $0.17 \pm 0.10$ & 8.45 & N93* $^{*}$ \\
\hline
\end{tabular}

Notes to Table 2:

$\mathbf{0 0 2 8}+\mathbf{0 8 1} \equiv$ Holmberg 11. Confusion. The BCG is a member of the Hickson compact group No. 2B (Hickson 1982). Three galaxies in beam. The nearest one is UGC 312, an Sc galaxy 0.7 mag brighter at $5^{\mathrm{s}}$ to the East and $27^{\prime \prime}$ to the South with $V=4335 \mathrm{~km} \mathrm{~s}^{-1}$

$\mathbf{0 1 2 7}+\mathbf{3 0 7 A}$ - Confusion? galaxy $0.5 \mathrm{mag}$ brighter, at $2.3^{\mathrm{s}}$ to the East and $4^{\prime}$ to the North, with no known velocity.

$\mathbf{0 1 3 6}+\mathbf{3 2 8}$ - Confusion? Scd galaxy KUG $0136+329,0.5$ mag fainter, at $3.6^{\mathrm{s}}$ to the West and $6^{\prime}$ to the North, with no known velocity.

$\mathbf{0 2 0 1}+\mathbf{2 8 4} \equiv$ KUG $0201+284$. No confusion. Uncertain parameters. In group with the SO galaxy NGC 805 at $11^{\mathrm{s}}$ to the East and $9.3^{\prime}$ to the North with $V=4573 \mathrm{~km} \mathrm{~s}{ }^{-1}$ and with NGC 807 at $21^{\prime}$ with $V=4764 \mathrm{~km} \mathrm{~s}^{-1}$.

$\mathbf{0 2 0 7}-\mathbf{0 8 0} \equiv$ KUG $0207-080$. Confusion by E-S0 galaxy NGC 842 at $1.3^{\mathrm{s}}$ to the East and $1^{\prime}$ to the North, at $V=3836 \mathrm{~km} \mathrm{~s}^{-1}$ ? No beam correction was made

0310-054 $\equiv$ KUG 0310-054. Confusion? S0 galaxy NGC 1248, 2.5 ${ }^{\mathrm{m}}$ brighter, at $1.1^{\mathrm{s}}$ to the East and $2.7^{\prime}$ to the North with $V=2209 \mathrm{~km} \mathrm{~s}^{-1}(17 / \mathrm{h} \mathrm{kpc}$ in projection)

0335-057A - Confusion? Scd galaxy PGC 13407, 4 mag brighter, no velocity, at $6^{\mathrm{s}}$ to the East and $8^{\prime}$ to the South.

0743+591B - Confusion. Irr galaxy $14.7 \mathrm{mag}$ at $114^{\mathrm{s}}$ to the East and $16^{\prime \prime}$ to the North, $V=6420 \mathrm{~km}^{\prime-1}$.

0750+603AB - Pair of BCGs with $\Delta V \sim 200 \mathrm{~km} \mathrm{~s}^{-1}$, separated by $3^{\mathrm{s}}$ in the EW direction and $32^{\prime \prime}$ in the SN direction, corresponding to a projected distance of $24 / \mathrm{h}$ kpc. Not resolved by the single-dish telescope beam.

$\mathbf{0 7 5 5}+\mathbf{5 8 7} \equiv$ MCG $10-12-47$. Flux corrected by $25 \%$ due to a large offset in the pointing. Confusion with MCG $10-12-51$ (三 KUG $0755+588)$ at $17^{\mathrm{s}}$ to the East and $5.7^{\prime}$ to the North, at $V=5760 \mathrm{~km} \mathrm{~s}^{-1}$.

$\mathbf{0 7 5 6}+\mathbf{6 1 1}$ - No confusion. Uncertain parameters.

0814+579A - Confusion with the BCG SBS $0814+579 \mathrm{~B}$ with $V=7918 \mathrm{~km} \mathrm{~s}^{-1}, 2.2^{\mathrm{s}}$ to the East, $1.1^{\prime}$ to the North, and faint galaxy at $5.1^{\mathrm{s}}$ to the West

$\mathbf{0 8 4 4}+\mathbf{7 0 3} \equiv$ KUG $0844+703$. In group. Confused by Sm galaxy UGC 4593 at $V=3626 \mathrm{~km} \mathrm{~s}^{-1}$ at $5.1^{\prime}$ and NGC 2650 at $V=3826 \mathrm{~km} \mathrm{~s}{ }^{-1}$ at $8^{\prime} . F(\mathrm{NGC} 4953)=$ $4.66 \mathrm{Jy} \mathrm{km} \mathrm{s}^{-1}$ and $W_{50}=241 \mathrm{~km} \mathrm{~s}^{-1}$ (Mebold et al. 1979), in good agreement with our data

$\mathbf{0 9 1 2}+\mathbf{5 9 9}$ - Interacting galaxies in contact.

0926+606 A and B - Pair of BCGs of similar brightnesses, separated by $2.5^{\mathrm{s}}$ in the EW direction and by $1.2^{\prime}$ in the SN direction, corresponding to $14 / \mathrm{h}$ kpc in projection $\Delta V \sim 100 \mathrm{~km} \mathrm{~s}^{-1}$. We have modeled the two peak HI-profile to derive parameters for each component of the pair. For 0926+606A $\left(\mathrm{RA}(1950)=09^{\mathrm{h}} 26^{\mathrm{m}} 20.1^{\mathrm{s}}, \mathrm{DEC}(1950)\right.$ $\left.=60^{\circ} 40^{\prime} 02^{\prime \prime}\right), V(\mathrm{HI})=4090 \mathrm{~km} \mathrm{~s}^{-1}, W_{50}=78 \pm 23 \mathrm{~km} \mathrm{~s}^{-1}, W_{20}=120 \pm 37 \mathrm{~km} \mathrm{~s}^{-1}$, and $\log M(\mathrm{HI})=9.04 ;$ For 0926+606B $\left(\mathrm{RA}(1950)=09^{\mathrm{h}} 26^{\mathrm{m}} 23.0^{\mathrm{s}}, \mathrm{DEC}(1950)=\right.$ $\left.60^{\circ} 41^{\prime} 15^{\prime \prime}\right), V(\mathrm{HI})=4002 \mathrm{~km} \mathrm{~s}^{-1}, W_{50}=67 \pm 23 \mathrm{~km} \mathrm{~s}^{-1}, W_{20}=120 \pm 37 \mathrm{~km} \mathrm{~s}^{-1}$, and $\log M(\mathrm{HI})=8.96$.

1011+601 - Paired with another BCG (SBS 1011+600) which is also included in the statistical sample. The separation is $1.0^{\mathrm{s}}$ in the EW direction and $3^{\prime}$ in the NS direction, corresponding to $20 / \mathrm{h} \mathrm{kpc}$ in projection. The pair is not resolved by the single-dish beam.

$\mathbf{1 0 2 8}+\mathbf{5 6 6}$ - No confusion. Uncertain parameters.

$\mathbf{1 0 5 4 + 5 0 4}$ - No confusion. Uncertain parameters.

1057+511A $\equiv$ UGC 6074. Sdm galaxy MCG 9-18-67, 1.3 mag fainter, at $2.8^{\mathrm{s}}$ to the West, $4.3^{\prime}$ to the South, with a velocity of $230 \pm 100 \mathrm{~km} \mathrm{~s} \mathrm{~s}^{-1}$ larger $(6 \mathrm{~m}$ data). Physical pair $\left(45 \mathrm{kpc} / \mathrm{h}\right.$ in projection). Armstrong \& Wooten (1986) obtained $F=2.2 \mathrm{Jy} \mathrm{km} \mathrm{s}{ }^{-1}$

$\mathbf{1 1 1 4 + 5 1 7}$ - Confusion with Sb galaxy UGC 6309, $2.7 \mathrm{mag}$ brighter, at $14.4^{\mathrm{s}}$ to the East and $3^{\prime}$ to the North with $V=2876 \mathrm{~km} \mathrm{~s}^{-1}$ and $F=4.35 \mathrm{Jy} \mathrm{km} \mathrm{s}{ }^{-1}$. $W_{50}=220 \mathrm{~km} \mathrm{~s}{ }^{-1}$, $W_{20}=264 \mathrm{~km} \mathrm{~s}^{-1}$ (Haynes \& Giovanelli 1991). Pair? 40/h kpc in projection.

$\mathbf{1 1 1 4}+\mathbf{5 1 4 5} \equiv \mathrm{UGC} 6309$, not a BCG. See the note for SBS $1114+5145$.

$\mathbf{1 1 2 3}+\mathbf{5 7 6}-$ No confusion. Uncertain parameters.

$\mathbf{1 1 2 5}+\mathbf{5 6 2}$ - Flux corrected by $42 \%$ due to offset of pointing relative to correct position. Possible confusion by Sc galaxy MCG $9-19-82$ at $22.6^{\mathrm{s}}$ to the West, $63^{\prime \prime}$ to the South $\left(13.1^{\mathrm{s}}\right.$ from telescope position). No known radial velocity

$\mathbf{1 1 2 5}+\mathbf{5 2 4}$ - Possible confusion with MCG 9-19-85, at $9.5^{\mathrm{s}}$ to the East and 4.1' to the North; unknown velocity. 
Notes to Table 2 (continued):

$\mathbf{1 1 2 9 + 5 7 6}$ - Confusion with a close Irr galaxy (MCG 10-17-10) 1.7 mag brighter, $1.3^{\mathrm{s}}$ to the East, $3.8^{\prime}$ to the North, $V=1296 \mathrm{~km} \mathrm{~s} \mathrm{~s}^{-1}$. Pair? $(15 / \mathrm{h} \mathrm{kpc}$ in projection)

1136+607 - Confusion with Sc galaxy UGC 6619, 2.6 mag brighter, at $19^{\mathrm{s}}$ to the East and $2^{\prime}$ to the North, with $V(\mathrm{HI})=3465 \mathrm{~km} \mathrm{~s}^{-1}, F=10.9 \mathrm{Jy} \mathrm{km} \mathrm{s}{ }^{-1}, W_{50}=279 \mathrm{~km} \mathrm{~s}^{-1}$, $W_{50}=327 \mathrm{~km} \mathrm{~s}^{-1}$ (Haynes \& Giovanelli 1991). Pair? (28/h kpc in projection). Also a close companion, a galaxy of the same brightness at $4.1^{\mathrm{s}}$ to the West.

$\mathbf{1 1 3 7 + 5 8 9}$ - Uncertain parameters. No confusion. Galaxy $0.5 \mathrm{mag}$ fainter at $15.5^{\mathrm{s}}$ to the West and $2^{\prime}$ to the South, with $V=25440 \mathrm{~km} \mathrm{~s}{ }^{-1}$.

1144+591 No confusion. MCG 10-17-56, 1.2 mag brighter, at $8^{\mathrm{s}}$ to the West and $9^{\prime}$ to the South, at $V \sim 3500 \mathrm{~km} \mathrm{~s}^{-1}$ (6 m data). In group or pair? UGC 6732 with $V=$ $2285 \mathrm{~km} \mathrm{~s}^{-1}$, at $14^{\prime}(\sim 80 / \mathrm{h} \mathrm{kpc} \mathrm{in} \mathrm{projection).}$

$\mathbf{1 1 4 7 + 5 2 0}$ - Confusion with the Sc UGC 6802 at $13.9^{\mathrm{s}}$ to the East and $7^{\prime}$ to the North with $V=1253 \mathrm{~km} \mathrm{~s}^{-1}$

$\mathbf{1 1 4 9 + 5 9 6}$ - No confusion. In group. Inside $30^{\prime}$ radius: NGC $3894\left(V=3242 \mathrm{~km} \mathrm{~s}^{-1}\right)$ and NGC $3895\left(V=3129 \mathrm{~km} \mathrm{~s}^{-1}\right)$.

$1159+516 \mathrm{~B}-$ Possible confusion by faint galaxy at $\sim 5^{\prime}$ to the North. Uncertain parameters.

$1216+460$ - Elongated edge-on spiral galaxy. Possible confusion with low-surface brightness galaxy at $1^{\prime}$ to the South and with unknown velocity. Pair?

$\mathbf{1 2 1 6}+\mathbf{5 5 1}-$ No confusion. Uncertain parameters.

1221+585 - Confusion? Large difference between optical and HI velocities. Sc galaxy $\left(a=30^{\prime \prime}\right)$ at $7.8^{\mathrm{s}}$ to the West and $11.5^{\prime}$ to the North. No velocity. In group: 3 bright galaxies inside $30^{\prime}$ radius with velocities near $4500 \mathrm{~km} \mathrm{~s}^{-1}$

$1221+545 B$ - No confusion. Uncertain parameters.

1222+614 - Confusion? Sab galaxy of similar brightness (MCG 10-18-43) at $8^{\mathrm{s}}$ to the West and $9.5^{\prime}$ to the South with no known velocity.

$\mathbf{1 2 4 0 + 5 5 4 C} \equiv$ UGC 7887. Confusion with SBb galaxy of the same brightness (NGC 4644) at $12^{\mathrm{s}}$ to the West with $V=4764 \mathrm{~km} \mathrm{~s} \mathrm{~s}^{-1}$. In group: 5 galaxies of similar brightnesses inside $30^{\prime}$ radius with velocities around $4800 \mathrm{~km} \mathrm{~s}^{-1}$

$\mathbf{1 2 4 2 + 5 4 9}$ - Confusion with the S galaxy NGC 4669, at $10^{\text {s }}$ to the East and $11^{\prime}$ to the North, with $V=4835 \mathrm{~km} \mathrm{~s}^{-1}$ (Bottinelli et al. 1999)

$\mathbf{1 3 1 4}+\mathbf{6 0 5}-$ No confusion. Uncertain parameters.

1317+523B $\equiv$ MCG 9-22-49. Confusion with the BCG SBS 1317+523A, which is at $20^{\mathrm{s}}$ to the West and $1.5^{\prime}$ to the North, and has the same HI velocity $\left(V=4619 \mathrm{~km} \mathrm{~s}{ }^{-1}\right)$ and

HI line widths. After subtraction of the integrated flux density contribution from SBS $1317+523 \mathrm{~A}$, we find $F=3.7 \mathrm{Jy} \mathrm{km} \mathrm{s}^{-1}$. In a group with SBS $1317+523 \mathrm{~A}$, MCG $9-22-48$ and SBS $1318+520$ at $11.4^{\mathrm{s}}$ to the East and $17^{\prime}$ to the South, with $V=4740 \mathrm{~km} \mathrm{~s}^{-1}$ (see the note for SBS $1317+523 \mathrm{~A}$, in Table 1).

$\mathbf{1 3 1 9 + 5 7 9 A B} \equiv$ NGC 5113, edge-on Sbc with two HII regions at the ends (A and B). Confusion with Sc galaxy NGC 51091.7 mag brighter at $29^{\mathrm{s}}$ to the West and $2.7^{\prime}$ to the South with $V=2131 \mathrm{~km} \mathrm{~s}^{-1}$. Possible confusion with MCG 10-19-63 at $12^{\prime}$ to the North. Unknown velocity.

$\mathbf{1 3 4 0 + 5 2 9}$ - Paired with MK 1481, a BCG which is 0.5 mag fainter, at $8^{\mathrm{s}}$ to the East and $1.2^{\prime}$ to the South, with $V=1880 \pm 78 \mathrm{~km} \mathrm{~s}^{-1}$. The Green Bank flux of the pair is a factor of 1.6 larger than that measured at Nançay. No confusion.

1341+529 - Paired with MK 1480, a BCG 0.5 mag brighter, at $8^{\mathrm{s}}$ to the West and $1.2^{\prime}$ to the North, with $V=1847 \pm 48 \mathrm{~km} \mathrm{~s}^{-1}$. No confusion.

$1401+490$ - Confusion? There are another compact galaxy of about the same brightness at $1.6^{\prime}$ to the South, and a slightly brighter galaxy at $4.5^{\mathrm{s}}$ to the West and $2^{\prime}$ to the North. Unknown velocities.

$\mathbf{1 4 5 0}+\mathbf{4 3 9}$ 三 UGC 9569. Confusion with UGC $9567\left(a(25)=60^{\prime \prime}\right)$ at $13.5^{\mathrm{s}}$ to the West and $4.3^{\prime}$ to the South with $V=2504 \pm 5 \mathrm{~km} \mathrm{~s}{ }^{-1}$. ( $\sim 30 / \mathrm{h} \mathrm{kpc}$ in projection). Interaction? Schneider et al. (1992) give $F=10.0 \mathrm{Jy} \mathrm{km} \mathrm{s}{ }^{-1}, W_{50}=154 \mathrm{~km} \mathrm{~s}^{-1}, W_{50}=183 \mathrm{~km} \mathrm{~s}^{-1}$. We have attempted a decomposition to estimate fluxes for each galaxy: $F$ (UGC 9569 ) $=4.9 \pm 0.8 \mathrm{Jy} \mathrm{km} \mathrm{s}^{-1}, F(\mathrm{UGC} 9567)=5.9 \pm 1.0 \mathrm{Jy} \mathrm{km} \mathrm{s}^{-1}$

$\mathbf{1 5 2 3 + 5 1 9}$ - No confusion, uncertain parameters.

$\mathbf{1 5 3 3 + 5 7 4} \equiv$ VII Zw 611 . Confusion? Pair or two components in the process of merging? Difference in optical velocities is about $150 \mathrm{~km} \mathrm{~s}^{-1}$. Sc galaxy MCG $10-22-21,0.5$ mag brighter, at $12^{\mathrm{s}}$ to the West and $4^{\prime}$ to the North. No known velocity.

$1533+469$ - Pair or interacting system, Galaxy 1.5 mag fainter at $2.2^{\mathrm{s}}$ to the West and $18^{\prime \prime}$ to the North. No confusion.

$\mathbf{1 5 4 2}+\mathbf{5 7 3 B} \equiv \mathrm{UGC} 10002$. SBc galaxy. Interacting system with the BCG $1542+573 \mathrm{~A}$. In group. Sc galaxy UGC 10022 with $V=3957 \mathrm{~km} \mathrm{~s}^{-1}$ and galaxy MCG+10-22-36 inside $30^{\prime}$ radius. The flux is highly uncertain due to a large correction for pointing offset.

$\mathbf{1 5 5 5 + 5 1 5}$ - Confusion? Optical and radio velocities differ by $\Delta V=200 \mathrm{~km} \mathrm{~s}^{-1}$. Sc galaxy $\left(a(25)=1^{\prime}\right), 2$ mag brighter, at $1.1^{\mathrm{s}}$ to the East and $7^{\prime}$ to the North. No known velocity. In group? Sab galaxy at $V=3706 \mathrm{~km} \mathrm{~s}^{-1}$ inside $30^{\prime}$ radius.

$\mathbf{1 5 5 8 + 5 8 5}$ - Pair with SBS $1559+585$ (三 MCG 10-23-15), 2 mag brighter, at $16.5^{\mathrm{s}}$ to the East and $17^{\prime \prime}$ to the North (26/h kpc in projection). Parameters are given for the decomposed profile.

$\mathbf{1 6 3 2}+\mathbf{5 7 8}$ - No confusion. Uncertain parameters. The $V=4900 \mathrm{~km} \mathrm{~s}^{-1}$ feature may be due to some faint radio interference, or may be associated with a brighter galaxy at $\sim 0.9^{\prime}$ to the East and $3^{\prime}$ to the North.

$\mathbf{2 3 0 4 + 0 9 7}$ - No confusion. Uncertain parameters.

$\mathbf{2 3 1 1 + 2 3 5}$ - No confusion. Uncertain parameters. 
Table 3. HI non-detections of BCGs

\begin{tabular}{|c|c|c|c|c|c|c|}
\hline \multirow[t]{2}{*}{ IAU name } & \multirow{2}{*}{$\begin{array}{c}\text { Other } \\
\text { name or } \\
\text { prefix }\end{array}$} & \multicolumn{2}{|c|}{ Coord. (1950.0) } & \multirow{2}{*}{$\begin{array}{l}V(\text { opt }) \\
\mathrm{km} \mathrm{s}^{-1}\end{array}$} & \multirow{2}{*}{$\begin{array}{l}\text { RMS } \\
\text { mJy }\end{array}$} & \multirow{2}{*}{$\begin{array}{l}\text { Run } \\
\text { Note }\end{array}$} \\
\hline & & R.A. & Dec. & & & \\
\hline 1 & 2 & 3 & 4 & 5 & 6 & 7 \\
\hline $0109-019$ & MK563 & $\begin{array}{lll}01 & 09 & 16.9\end{array}$ & -015515 & 4900 & 2.0 & N94 \\
\hline $0155+021$ & MK1169 & 015503.9 & 021050 & 4950 & 5.8 & N93* \\
\hline $0200+023$ & MK585 & 020055.2 & 021935 & 6400 & 6.3 & G92* \\
\hline $0223+009$ & MK1038 & 022353.9 & 005611 & 1500 & 1.7 & N94* \\
\hline $0323-003$ & MK611 & 032340.9 & -002239 & 9095 & 5.7 & G92* \\
\hline $0333-048$ & MK613 & 033357.6 & -045156 & 6490 & 7.5 & G92 \\
\hline $0715+594$ & SBS & 071519.0 & 592655 & 2400 & 2.1 & N94* \\
\hline $0745+587$ & SBS & 074524.6 & 584711 & 6370 & 2.9 & N93* \\
\hline $0749+582$ & SBS & 074942.0 & 581600 & 9424 & 1.9 & N93 \\
\hline $0807+580 B$ & SBS & 080804.3 & 580325 & 7980 & 6.4 & G92 \\
\hline $0810+583$ & SBS & 081011.2 & 582104 & 7786 & 6.0 & G92 \\
\hline $0907+593$ & SBS & 090715.9 & $5918 \quad 12$ & 8894 & 8.4 & G92 \\
\hline $0911+472$ & SBS & 091113.1 & 471428 & 8130 & 5.3 & G92 \\
\hline $0943+543^{+}$ & SBS & 094340.8 & 541933 & 1660 & 5.0 & G92* \\
\hline $0948+532$ & SBS & 094816.7 & 531300 & 13960 & 2.8 & N95 \\
\hline $1030+583^{+}$ & MK1434 & 103056.3 & 581920 & 2400 & 2.7 & N94* \\
\hline $1057+511 \mathrm{~B}^{+}$ & SBS & 105710.5 & 510722 & 3060 & 7.3 & G92 \\
\hline $1118+578 B$ & SBS & 111839.1 & 575330 & 2163 & 3.8 & N93 \\
\hline $1122+575^{+}$ & SBS & 112212.8 & 573237 & 1200 & 1.7 & N94 \\
\hline $1128+573^{+}$ & SBS & 112828.9 & 572033 & 1480 & 6.5 & G92 \\
\hline $1135+581^{+}$ & MK1450 & 113551.3 & 580924 & 900 & 2.8 & G92, N93* \\
\hline $1137+589^{+}$ & SBS & 113748.3 & 585510 & 1850 & 4.6 & N94 \\
\hline $1159+545^{+}$ & SBS & 115929.0 & 543232 & 3600 & 2.8 & N94 \\
\hline $1212+493^{+}$ & SBS & 121206.0 & 492318 & 3800 & 6.7 & N92 \\
\hline $1222+588^{+}$ & SBS & 122229.1 & 584917 & 4722 & 3.0 & N93 \\
\hline $1307+563^{+}$ & SBS & 130741.5 & 561821 & 5200 & 2.1 & N94 \\
\hline $1354+597 \mathrm{~B}$ & SBS & 135402.1 & 594217 & 3000 & 5.3 & G92 \\
\hline $1420+544$ & CG413 & 142059.1 & 542742 & 6280 & 5.7 & G91 \\
\hline $1423+518^{+}$ & CG424 & 142341.1 & 514636 & 2140 & 7.6 & G92 \\
\hline $1446+595^{+}$ & SBS & 144620.6 & 593420 & 2120 & 7.0 & G92 \\
\hline $1453+526^{+}$ & CG577 & 145309.0 & 523754 & 3280 & 7.5 & G92 \\
\hline $1459+483$ & CG608 & 145944.2 & 481812 & 3100 & 8.2 & G92 \\
\hline $1503+531^{+}$ & CG618 & 150302.0 & 530654 & 5500 & 3.0 & N94* \\
\hline $1614+600$ & SBS & 161451.9 & 600259 & 9100 & 2.5 & N93* \\
\hline $1620+577^{+}$ & SBS & 162015.5 & 574445 & 5600 & 2.0 & N94 \\
\hline $1716+302$ & MK894 & 171632.4 & 301406 & 4400 & 2.5 & N96 \\
\hline $2325-024$ & MK1131 & 232527.0 & -022624 & 2580 & 7.2 & G92 \\
\hline
\end{tabular}

Notes to Table 3:

All optical velocities have been obtained with the $6 \mathrm{~m}$ telescope IPCS with a typical accuracy of $100-150 \mathrm{~km} \mathrm{~s}^{-1}$. They come either from Stepanian et al. 1993 (a-d), or Lipovetsky et al.'s unpublished catalog of BCGs.

$\mathbf{0 1 5 5 + 0 2 1} \equiv$ UM 151

$\mathbf{0 2 0 0}+\mathbf{0 2 3} \equiv \mathrm{UM} 391$

$\mathbf{0 2 2 3}+\mathbf{0 0 9} \equiv \mathrm{IC} 0225$

$\mathbf{0 3 2 3}-\mathbf{0 0 3} \equiv$ KUG $0323-003$

$\mathbf{0 7 1 5}+\mathbf{5 9 4} \equiv \mathrm{UGC} 3789$

$\mathbf{0 7 4 5}+\mathbf{5 8 7} \equiv \mathrm{KUG} 0745+587$

$\mathbf{0 9 4 3}+\mathbf{5 4 3} \equiv \mathrm{KUG} 0943+543$

$\mathbf{1 0 3 0}+\mathbf{5 8 3} \equiv$ KUG $1030+583$

$\mathbf{1 1 3 5}+\mathbf{5 8 1}$ - Observed in the G92 and N93 runs, with $F$ (peak) $=22 \pm 4.1 \mathrm{mJy}$, and $5.4 \pm 2.8$ mJy respectively. In both cases $V \sim 950 \mathrm{~km} \mathrm{~s}^{-1}$ as compared to $V(\mathrm{opt})=896 \pm 21 \mathrm{~km} \mathrm{~s}^{-1}$. This HI emission may come from a galaxy with similar brightness at $14^{\mathrm{s}}$ to the East and $45^{\prime \prime}$ to the South, which is entirely in the beam of the $140 \mathrm{ft}$ telescope but only partially in the beam of the Nançay telescope.

$\mathbf{1 5 0 3}+\mathbf{5 3 1}$ - Elongated edge-on S galaxy. Uncertain parameters.

$\mathbf{1 6 1 4}+\mathbf{6 0 0}$ - Confusion? Sc galaxy, 3 mag brighter. At $16.5^{\mathrm{s}}$ to the West and $1.8^{\prime}$ to the North. No known velocity. 

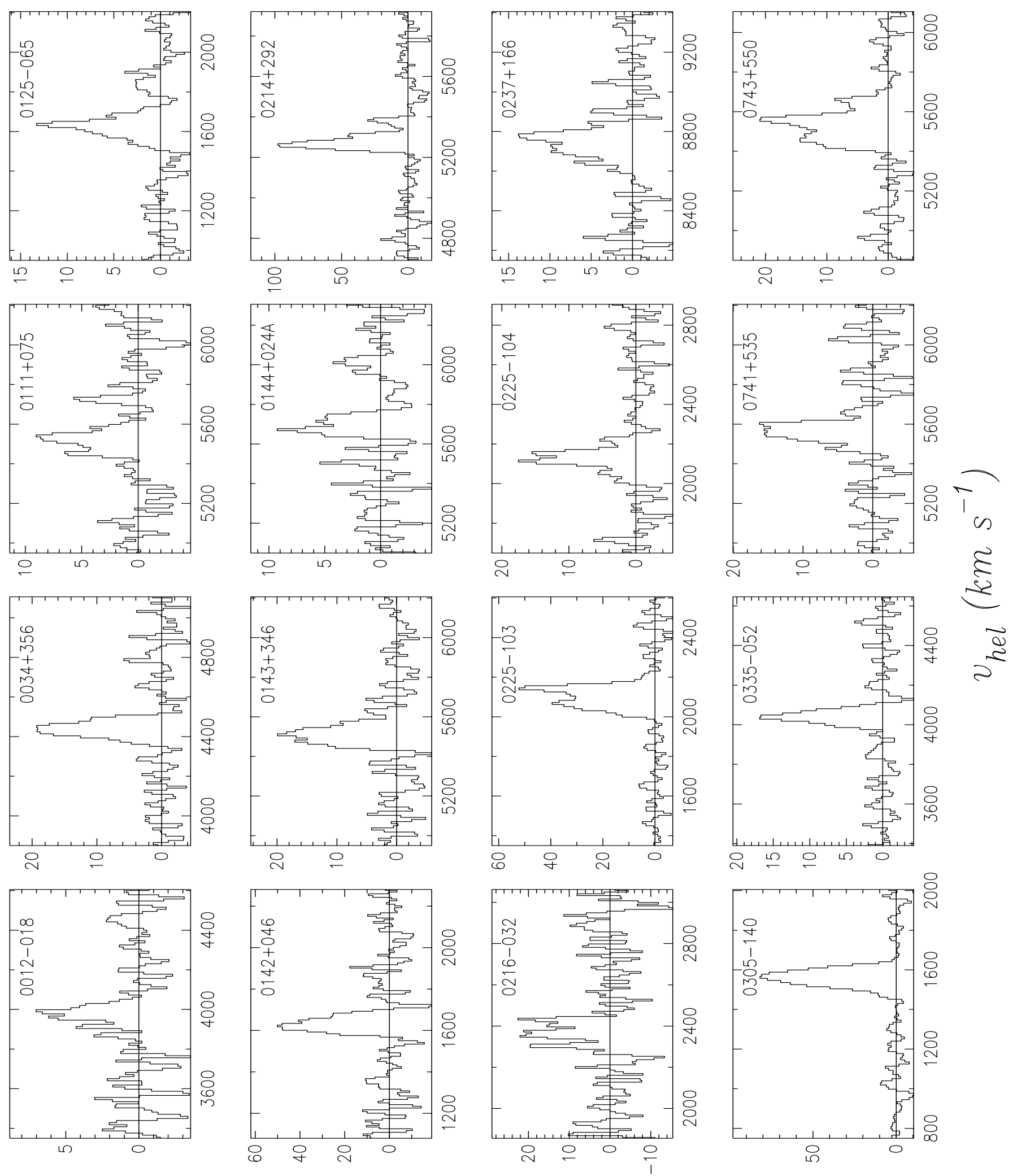

$$
\left(n_{\rho} u\right)^{n} \mathrm{~s}
$$

Fig. 1. HI profiles of the 79 certain BCG detections (Table 1). The ordinate is line flux density in mJy and the abscissa is heliocentric velocity in $\mathrm{km} \mathrm{s}^{-1}$ 
Trinh X. Thuan et al.: HI observations of blue compact galaxies
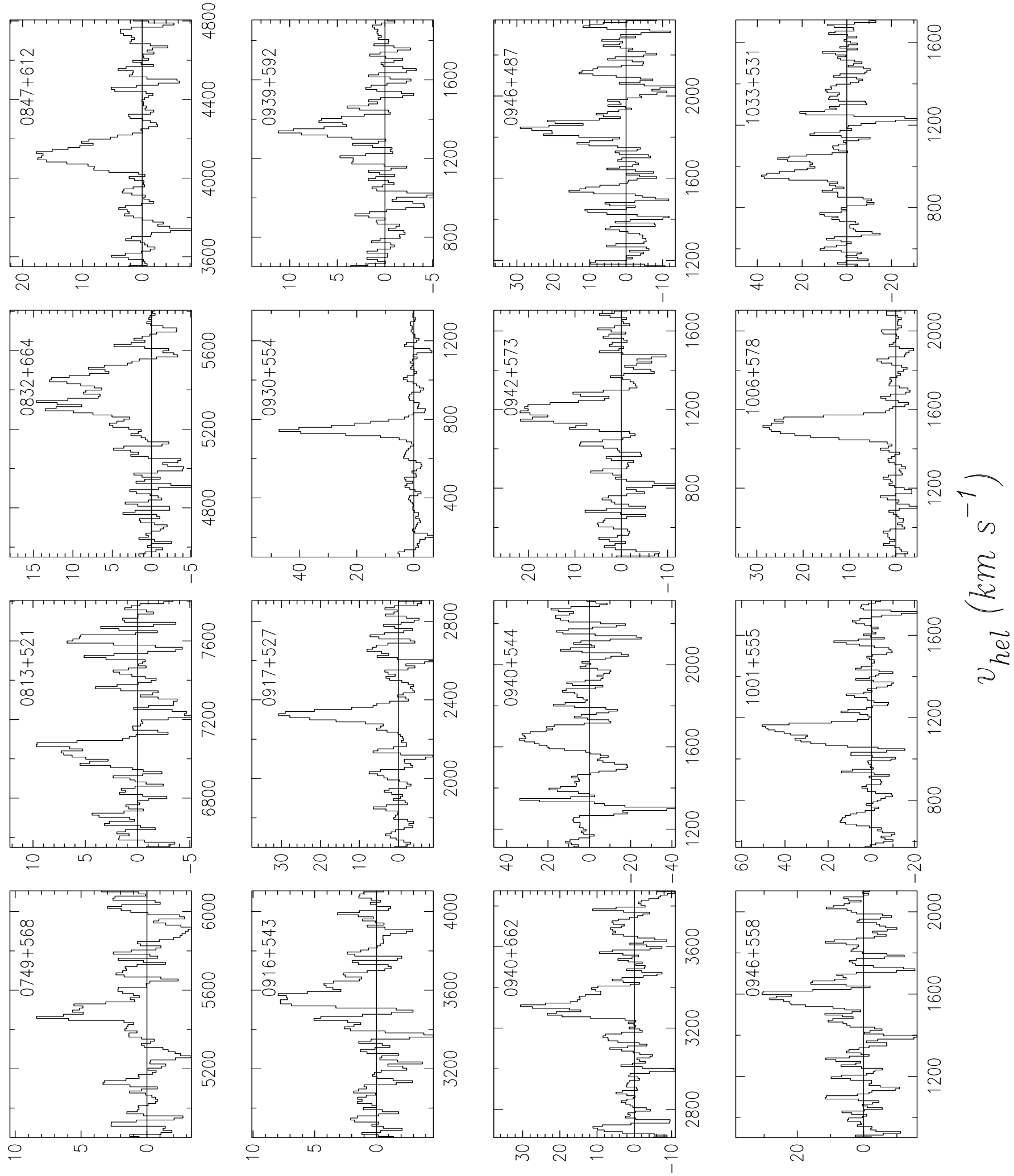

$$
\left(n_{\text {ruu }}\right)^{n} \mathrm{~s}
$$

Fig. 1. continued 

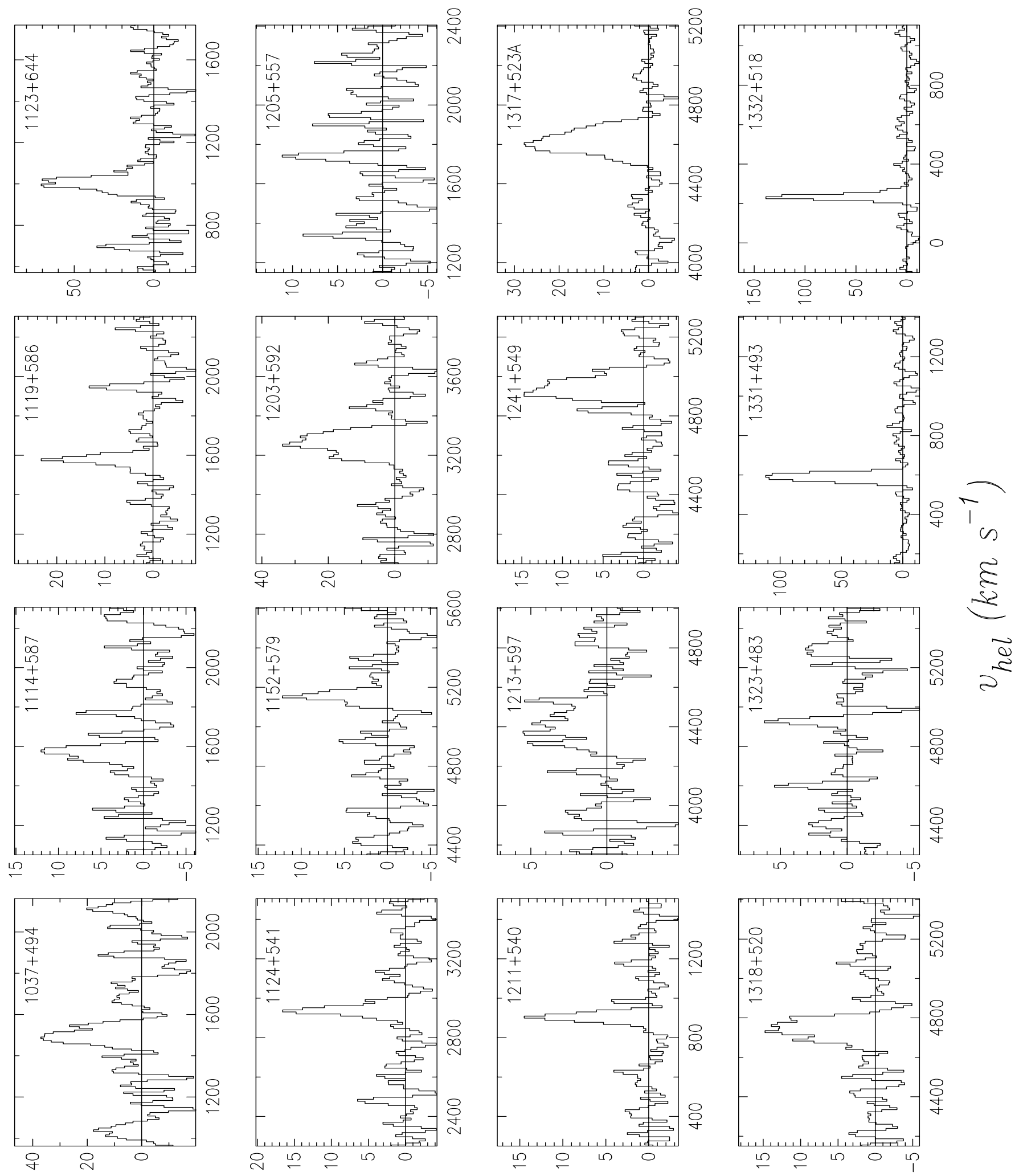

$$
\left(n_{\rho} u\right)^{n} \mathrm{~s}
$$

Fig. 1. continued 
Trinh X. Thuan et al.: HI observations of blue compact galaxies
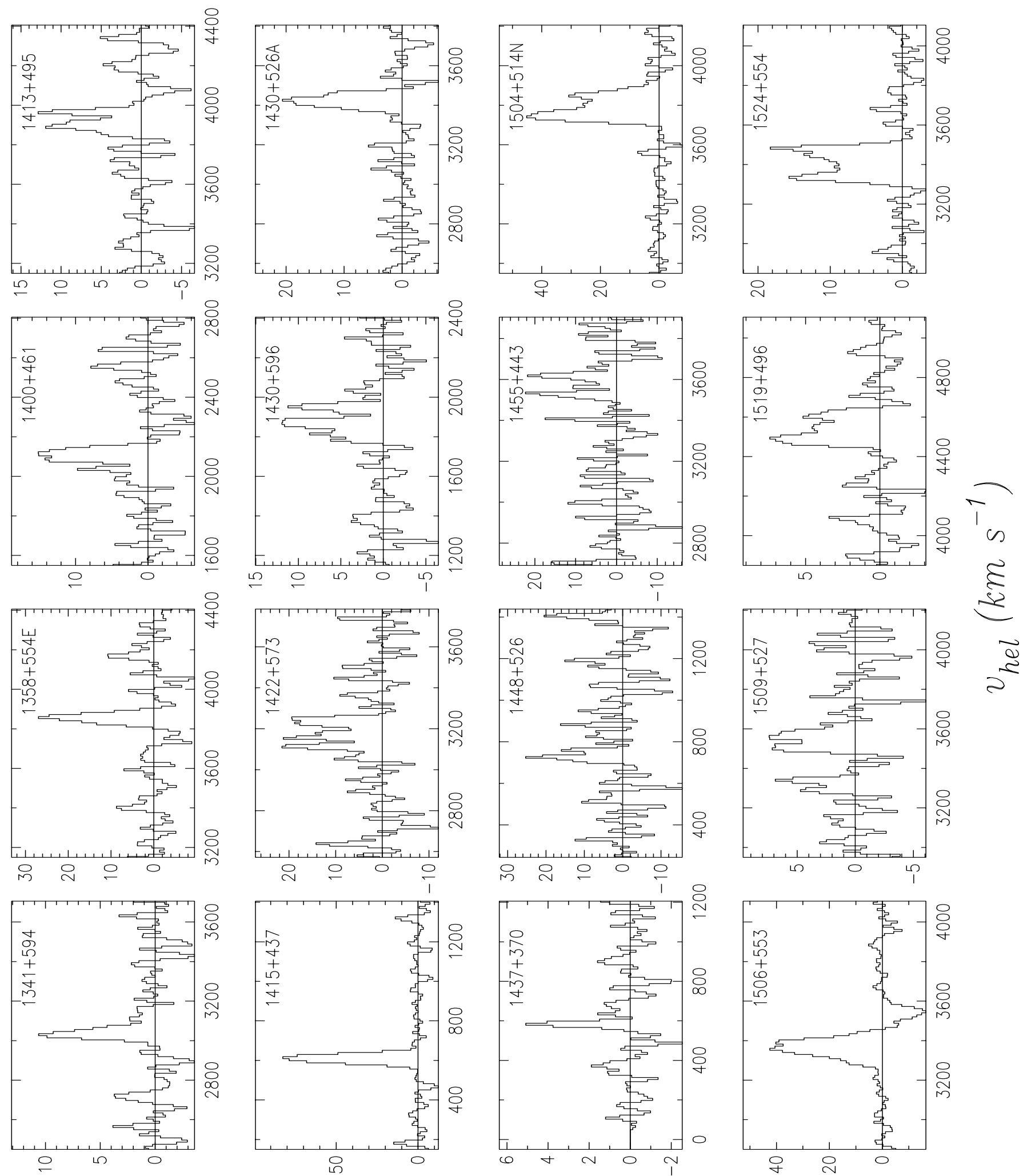

$\left(\right.$ nгul $^{n} \mathrm{~s}$

Fig. 1. continued 

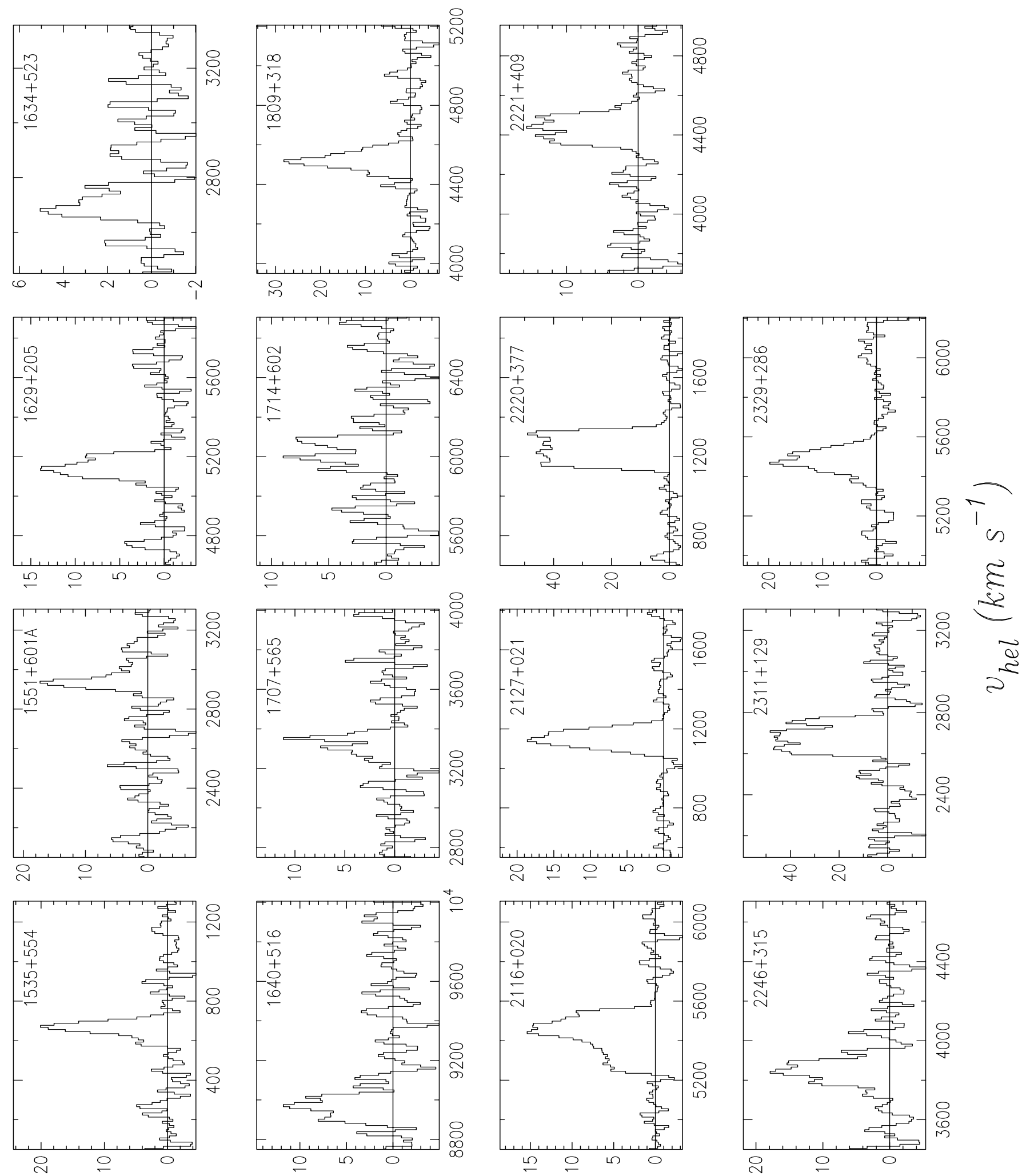

$\left(r_{\rho u}\right)^{n} S$

Fig. 1. continued 
Trinh X. Thuan et al.: HI observations of blue compact galaxies
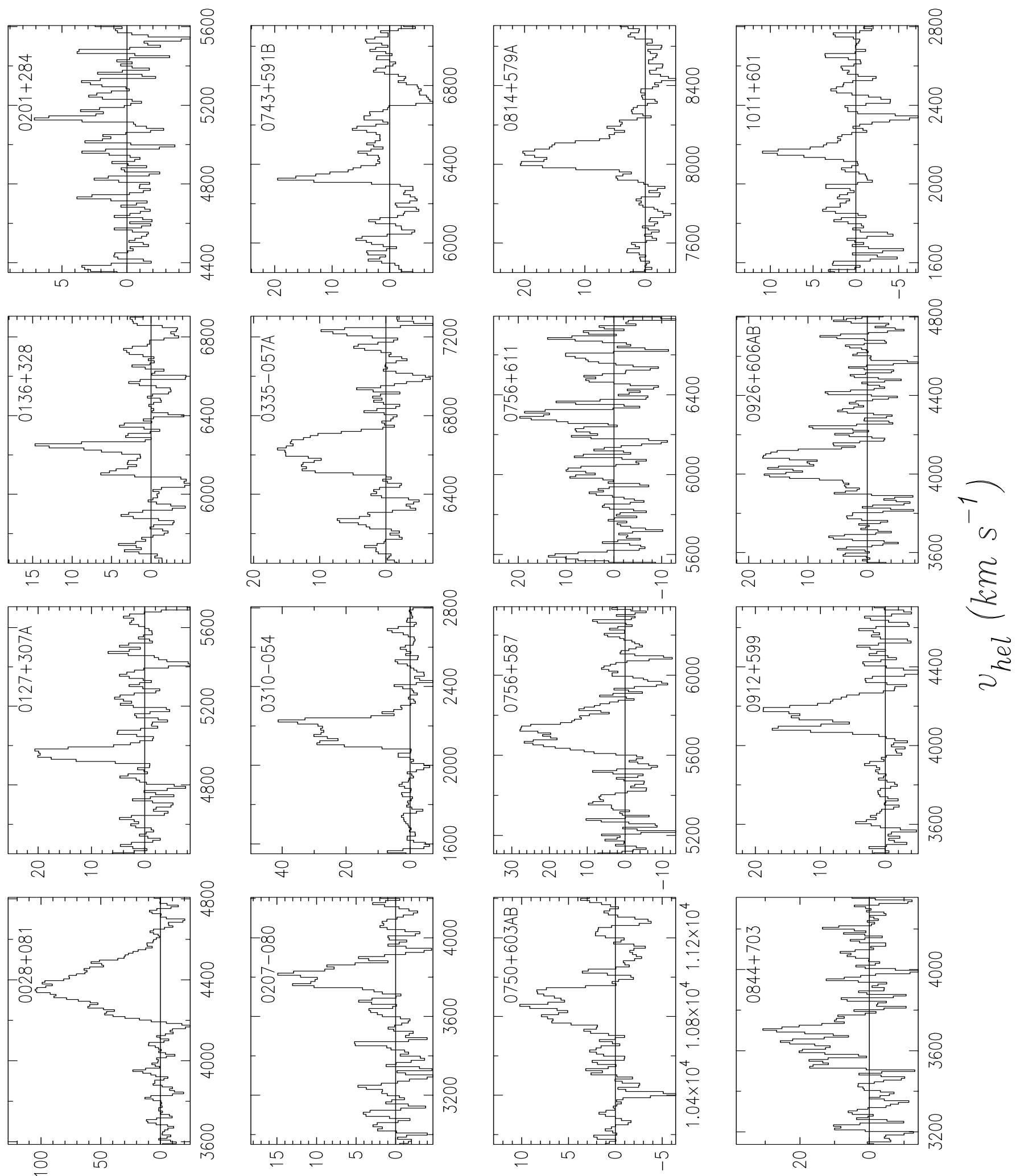

$$
\left(\hbar_{\Gamma} u\right)^{n} \mathrm{~S}
$$

Fig. 2. HI profiles of the 53 less secure or confused BCG detections (Table 2). The ordinate is line flux density in mJy and the abscissa is heliocentric velocity in $\mathrm{km} \mathrm{s}^{-1}$ 

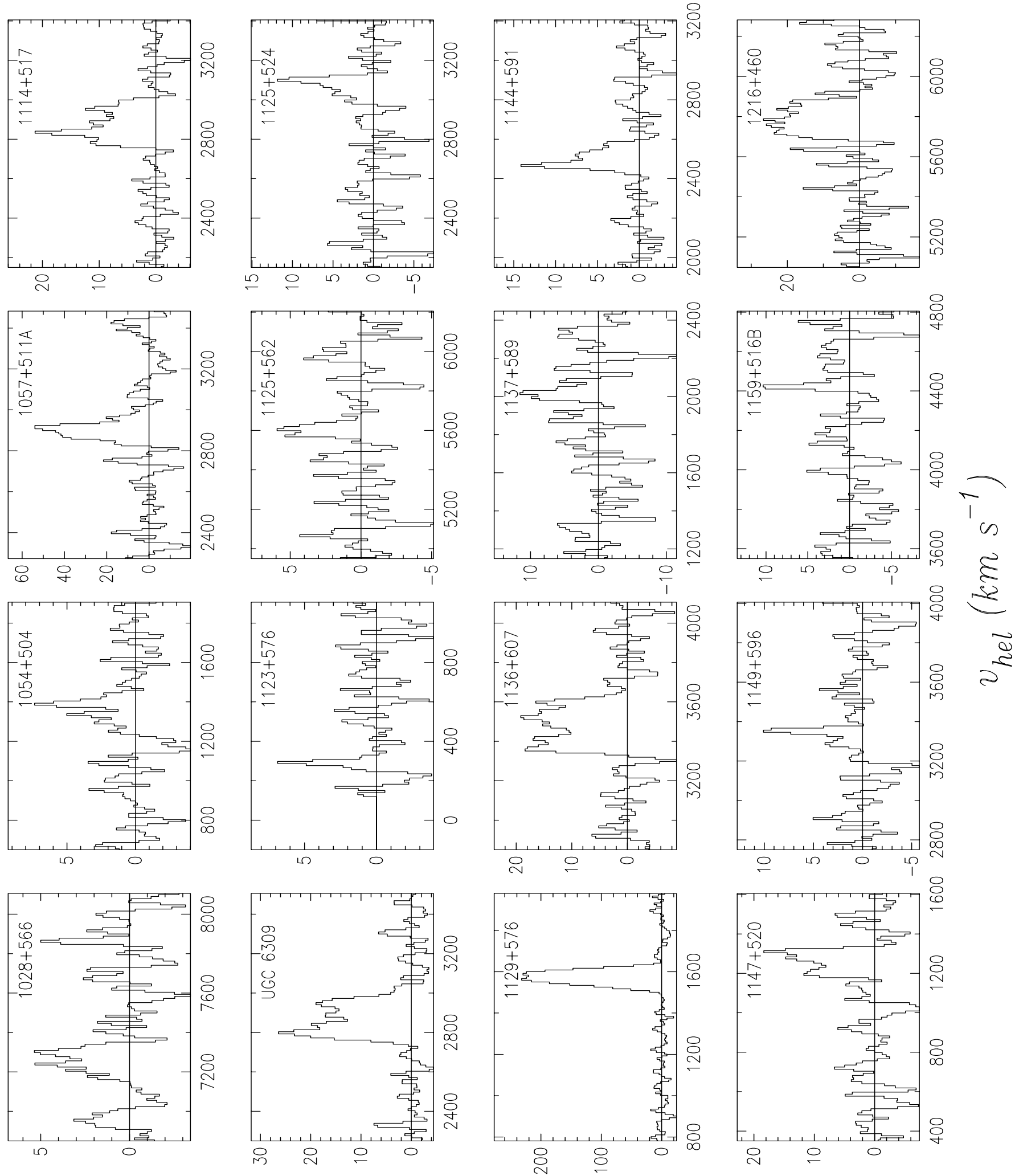

$$
\left(n_{\text {rul }}\right)^{n} \mathrm{~s}
$$

Fig. 2. continued 

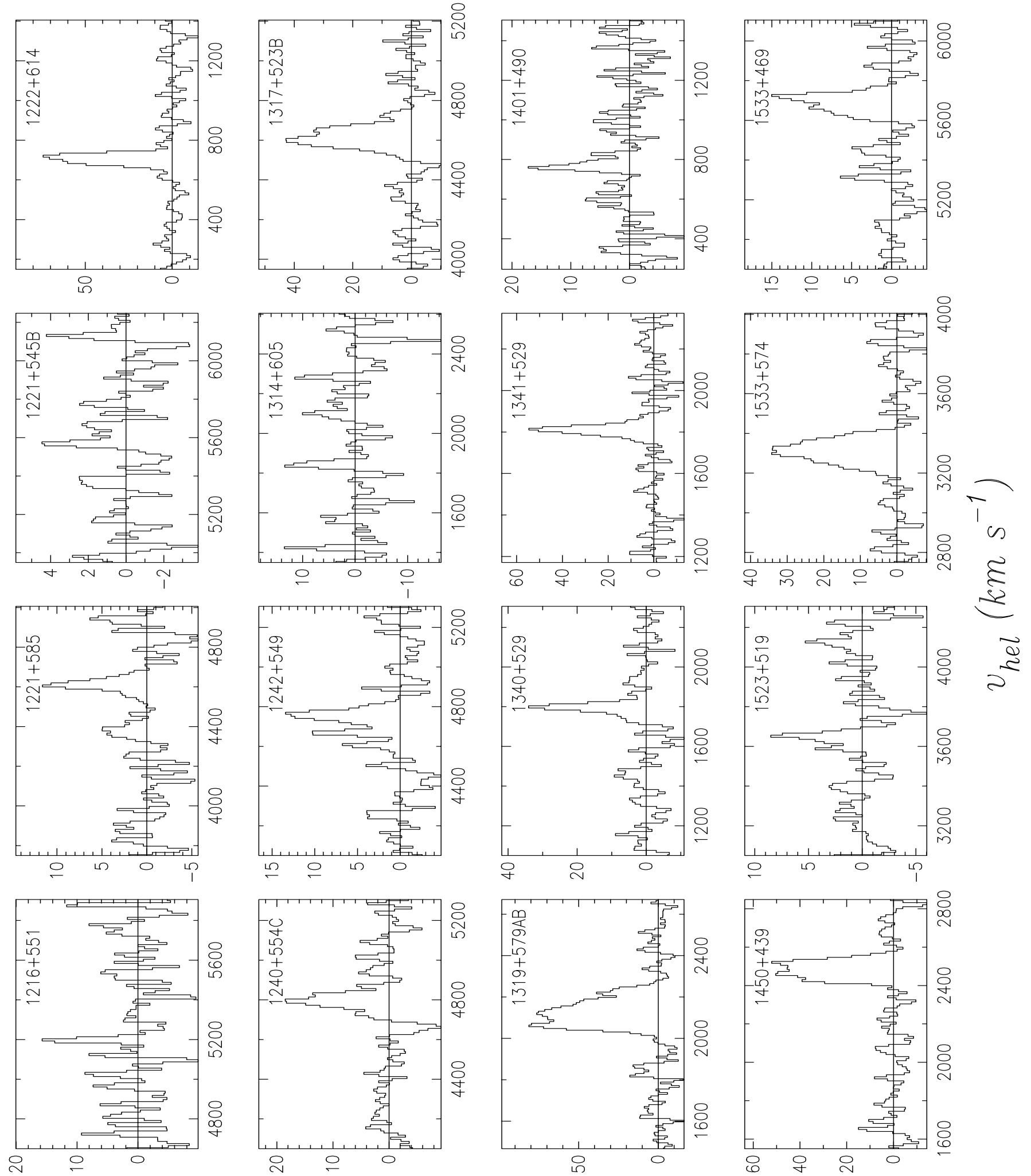

$$
\left(n_{\text {ruu }}\right)^{n} \mathrm{~s}
$$

Fig. 2. continued 

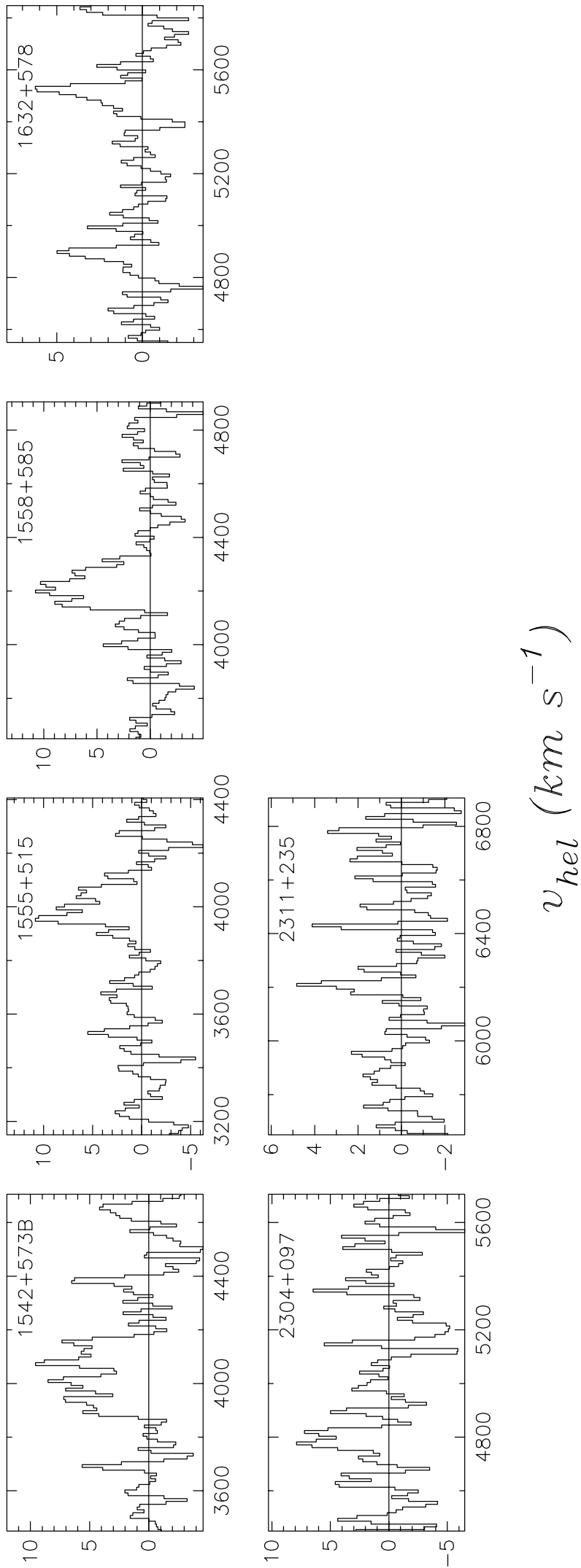

$$
(\text { n } u)^{n} \mathrm{~s}
$$

Fig. 2. continued
Column (5). Holmberg (H) major and minor diameters in arcsec to the limiting surface brightness of $B=$ $26.5 \mathrm{mag} \operatorname{arcsec}^{-2}$, derived mainly from CCD surface photometry (Lipovetsky et al. 1999b; Kniazev 1995). When CCD data was not available, Holmberg diameters were derived from measurements on the Palomar Sky Survey (PSS) blue prints and the relation obtained by leastsquare fitting to the diameter data from a subsample of 48 of our BCGs with CCD photometry:

$d_{\mathrm{H}}(\operatorname{arcsec})=2.53 d_{\mathrm{PSS}}(\operatorname{arcsec})+7.4^{\prime \prime}$.

Diameters derived in this way are marked by the letter e. They are less accurate than the CCD diameters and serve only to correct the HI fluxes for beam effects (Col. 12). We are in the process of deriving accurate CCD diameters for all BCGs in the statistical sample. Diameters are given only for BCGs observed at Nançay, which require a beam-flux correction (see Col. 12).

Column (6). Position angle in degrees of the major axis of the galaxy measured eastward from north.

Column (7). Heliocentric HI velocity in $\mathrm{km} \mathrm{s}^{-1}$ determined as the midpoint between the half-peak points on both sides of the HI profile. The velocity error is estimated from the peak signal-to-noise ratio as decribed by Schneider et al. (1986), and includes an estimate of errors based on the slope of the profile edges. It is calculated as: $\Delta V=1.5\left(W_{20}-W_{50}\right)(S / N)^{-1}$

where $S / N$ is the ratio of the peak signal to the rms noise.

Column (8). Distance $D$ in Mpc to the galaxy. We have used the Virgocentric flow model described by KraanKorteweg (1986) with a Virgocentric infall motion of $220 \mathrm{~km} \mathrm{~s}^{-1}$ and a Hubble constant of $75 \mathrm{~km} \mathrm{~s}^{-1} \mathrm{Mpc}^{-1}$.

Columns (9) and (10). Velocity widths in $\mathrm{km} \mathrm{s}^{-1}$ of the HI profile at $50 \%$ and $20 \%$ of peak. Again, errors are calculated following Schneider et al. (1986) and are based on the same method as for the velocities in Col. (7). The expected error for the $50 \%$ width is

$3.0\left(W_{20}-W_{50}\right)(S / N)^{-1}$ and

for the $20 \%$ width is

$4.7\left(W_{20}-W_{50}\right)(S / N)^{-1}$.

Column (11). Observed integrated flux density in Jy $\mathrm{km} \mathrm{s}^{-1}$. Following Schneider et al. (1990), we assume that the window over which the flux was measured is $20 \%$ wider than the velocity width measured at $20 \%$ of peak. The statistical error on the flux is then:

$\sigma=2\left(1.2 W_{20} / \Delta V\right)^{1 / 2} \Delta V \sigma_{V}$

where $\Delta V$ is the velocity resolution of the smoothed data, and $\sigma_{V}$ is the rms dispersion in the baseline measured at that resolution. The factor of 2 accounts for baseline uncertainties.

Column (12). HI flux densities corrected for telescope beam size. This correction is negligible for observations made with the NRAO $43 \mathrm{~m}$ telescope, as the FWHP of the telescope beam is $22^{\prime}$, much larger than the maximum 
size of our galaxies $\left(\leq 3^{\prime}\right)$. The same holds for the northsouth beam of the Nançay telescope which has a FWHP of $22^{\prime}$. But the east-west beam of the Nançay radio telescope has a FWHP of only $3.7^{\prime}$, and a beam correction needs to be applied. We follow Thuan \& Martin (1981) in assuming that the neutral gas is distributed like an elliptical Gaussian with half-power major and minor axis $a$ and $b$ in arcmin. Then:

$F_{\mathrm{c}}=F_{\mathrm{H}}\left[1+\left(a^{2} \sin ^{2} \mathrm{PA}+b^{2} \cos ^{2} \mathrm{PA}\right) / \theta^{2}\right]^{1 / 2}$

where $a$ and $b$ are the Holmberg diameters given in Col. (5), PA is the position angle in degrees given in Col. (6), and $\theta$ is the HPBW of the Nançay telescope in the East-West direction. The beam correction are generally small, between 1.01 and 1.03. For only a few BCGs does the correction factor reach $1.10-1.18$.

Column (13). Logarithm of the hydrogen mass $M_{\mathrm{H}}$ in solar masses calculated from:

$M_{\mathrm{H}}=2.36 \times 10^{5} F_{\mathrm{c}}\left(\mathrm{Jy} \mathrm{km} \mathrm{s}^{-1}\right) D^{2}\left(\mathrm{Mpc}^{2}\right)$

where $F_{\mathrm{c}}$ is the corrected flux density given in Col. (12) and $\mathrm{D}$ the distance to the galaxy given in Col. (8).

Column (14). Telescope used ( $\mathrm{G}=$ Green Bank, $\mathrm{N}=$ Nançay) and last two digits of the year of observation (The observation year ranges from 1992 to 1996). An asterisk in Table 1 means that there is additional information in the notes. All entries in Table 2 have supplemental notes.

Table 1 contains 79 entries with 34 galaxies in the statistical sample.

Table 2 contains 54 entries but only 53 are BCGs as $1114+5145 \equiv$ UGC6074 is not a BCG and has been observed to check for confusion with the BCG $1114+517 \equiv$ MK1445. Out of the 53 BCGs, we have determined that 32 BCGs are confused (see notes). The 21 non-confused BCGs in Table 2 are: $0201+284,0756+611,0912+599$, $1028+566,1054+504,1057+511 \mathrm{~A}, 1123+576,1137+589$, $1144+591,1149+596,1216+551,1221+545 \mathrm{~B}, 1314+605$, $1340+529,1341+529,1523+519,1533+469,1542+573 \mathrm{~B}$, $1632+578,2304+097$ and $2311+235$. They are marked by an asterisk in Col. 1. Table 2 contains 29 galaxies from the statistical sample, with 11 non-confused galaxies and 18 confused galaxies, among which are the pairs of BCGs 0926+606 A and B and 1011+600 and 1011+601 which are listed in a single entry.

Table 3 lists the BCGs which were not detected. It is organized as follows: the galaxy's IAU name (Col. 1), cross-reference to other names (Col. 2), its 1950 coordinates (Cols. 3 and 4 ). The heliocentric optical velocity on which the receiver was centered is given in Col. 5. The NRAO and Nançay receivers covered respectively a velocity range of 1350 and $4225 \mathrm{~km} \mathrm{~s}^{-1}$. Col. 6 lists the rms noise in mJy of the spectrum smoothed to a velocity resolution of $\sim 16 \mathrm{~km} \mathrm{~s}^{-1}$ for the Green Bank spectra and of $\sim 21 \mathrm{~km} \mathrm{~s}^{-1}$ for the Nançay spectra. Finally, Col. 7 gives the telescope used and the year of the observations. An asterisk signifies that more information can be found in the notes.

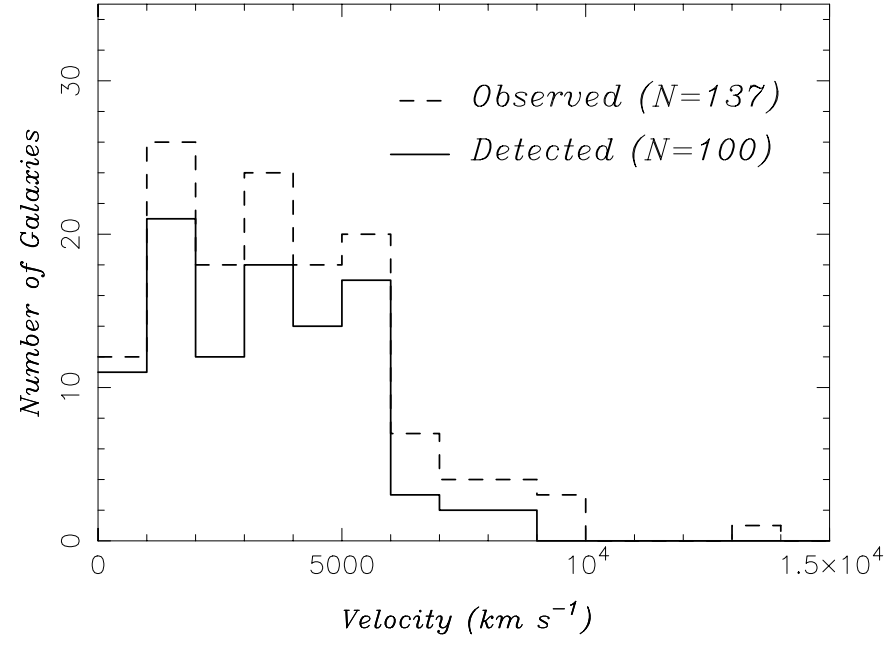

Fig. 3. Histogram of the number of all non-confused observed (dashed line) and detected (solid line) BCGs versus heliocentric velocity

Figures 1 and 2 show in order of increasing right ascension the HI profiles of the BCGs listed respectively in Tables 1 and 2. Several of the BCGs in Table 1 have previous HI measurements. The detailed references are given in the Notes section. Comparison of our measurements with those of previous authors is given in Appendix A.

\section{Discussion and summary}

We present here the HI observations of 79 galaxies of a statistical sample of 88 BCGs selected from the First and Second Byurakan objective prism surveys to have a HII region-like spectrum, an equivalent width of the [O III] $\lambda 5007$ line larger than $\approx 50 \AA$, and a heliocentric velocity less than or equal to $6000 \mathrm{~km} \mathrm{~s}^{-1}$. This statistical BCG sample shall be used in a subsequent paper to: 1) study the physical parameters governing the burst mode of star formation in dwarf galaxies; 2) study the chemical evolution of dwarf galaxies and 3) compare statistically the properties of BCGs with those of other types of dwarf galaxies. For comparison, we have also observed an additional 20 BCGs in the SBS zone with weaker emission lines, 47 BCGs with $V \leq 6000 \mathrm{~km} \mathrm{~s}^{-1}$ not in the SBS zone and 17 BCGs in the SBS zone with $V \geq 6000 \mathrm{~km} \mathrm{~s}^{-1}$, which are of particular astrophysical interest. For the statistical sample which contains 61 non-confused galaxies, we detected 34 BCGs with $S / N \geq 3-4$ (Table 1) and 11 with $2 \leq S / N \leq 3$ and no confusion (Table 2 ), giving a detection rate of $\approx 74 \%$. This is to be compared with the $80 \%$ detection rate of Thuan \& Martin (1981).

Figure 3 shows the histogram of all non-confused observed (dashed line) and detected (solid line) BCGs as a function of heliocentric velocity. It is seen that the detection rate is roughly constant, equal to $\approx 75 \%$, out to $6000 \mathrm{~km} \mathrm{~s}^{-1}$, so that the complete statistical sample does 


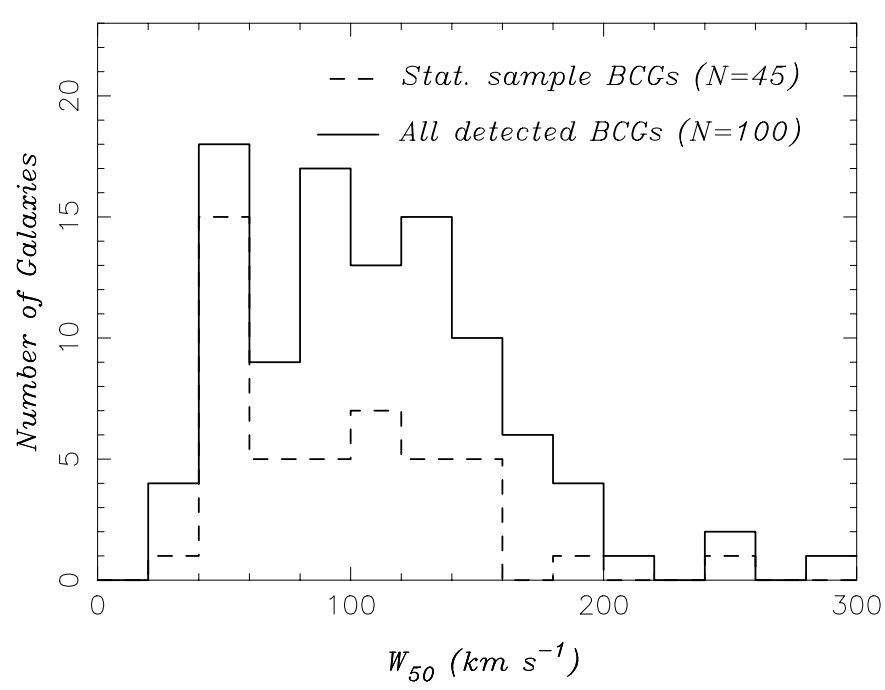

Fig. 4. Histogram of profile widths $\mathrm{W}_{50}$ for the 45 detected BCGs in the statistical sample (dashed line) and for the 100 detected BCGs in the enlarged sample (solid line)

not suffer from any bias due to telescope sensitivity drop off.

Figure 4 shows the distribution of $W_{50}$, the velocity width at half-maximum for both the statistical sample with $V \leq 6000 \mathrm{~km} \mathrm{~s}^{-1}$ (a total of 45 galaxies, dashed line) and all detected BGGs (a total of 100 galaxies, solid line). The peak at $\sim 50 \mathrm{~km} \mathrm{~s}^{-1}$ is due mainly to the statistical sample and may be slightly artificially enhanced as a third of the objects contributing to this peak are from Table 2 with lower signal-to-noise ratio profiles and hence with less reliable widths. Most of the statistical sample shows widths $\leq 160 \mathrm{~km} \mathrm{~s}^{-1}$. The mean width is $92 \pm 48 \mathrm{~km} \mathrm{~s}^{-1}$. These relatively small widths are characteristic of dwarf galaxies. The histogram of all detected BCGs shows a larger proportion of widths $\geq 60 \mathrm{~km} \mathrm{~s}^{-1}$ ) with a high end extending to $300 \mathrm{~km} \mathrm{~s}^{-1}$, implying rotational velocities comparable to those of large disk galaxies. Some of these high widths are contributed by more distant $\left(V \geq 6000 \mathrm{~km} \mathrm{~s}^{-1}\right)$ and hence more luminous and massive BCGs. Figure 5 shows the distribution of hydrogen masses for both the statistical (dashed line) and the enlarged sample of all detected BCGs (solid line). The HI masses for the statistical sample range between $410^{7} M_{\odot}$ and $510^{9} M_{\odot}$ with the distribution peaking at $\sim 310^{8} M_{\odot}$. The HI mass distribution for all detected BCGs extends from $\sim 10^{6} M_{\odot}$ to $10^{10} M_{\odot}$, with a broad peak at $\sim 10^{9} M_{\odot}$. The shifted peak toward higher HI masses is again partly due to more distant and more massive galaxies than in the statistical sample. BCGs are gas-rich objects, not a surprising conclusion, as gas is needed to fuel their very intense starforming activity. We have also shown in dashed-dotted lines the distribution of upper limits for $\mathrm{M}(\mathrm{HI})$ derived using the data in Table 3, and assuming that the HI flux is less than $2 \mathrm{rms} \times 100 \mathrm{~km} \mathrm{~s}^{-1}$. The HI mass upper limit distribution is very similar to the HI mass distribution of

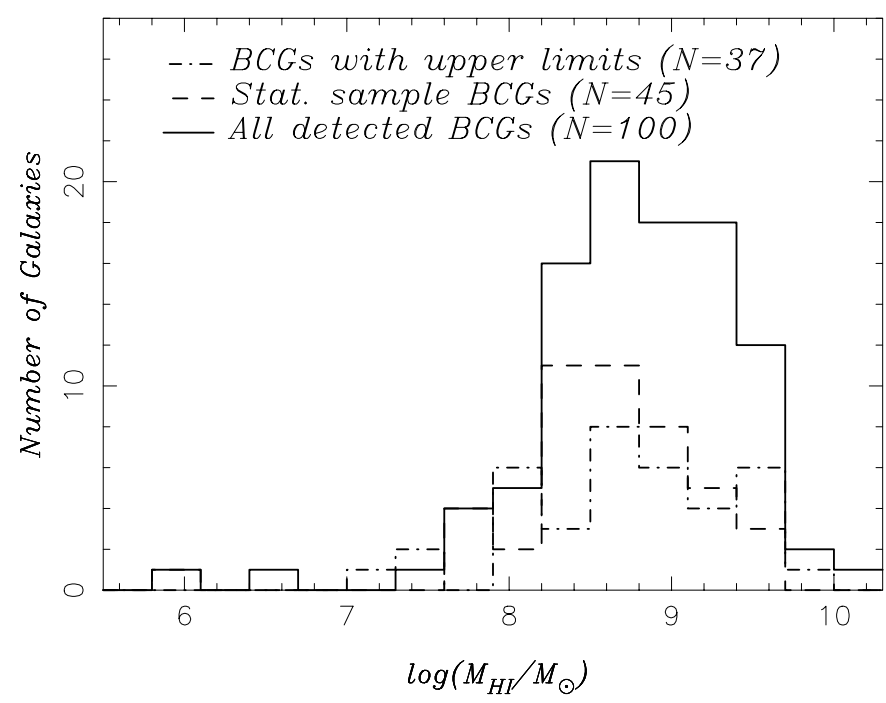

Fig. 5. Histogram of HI masses for the 45 detected BCGs in the statistical sample (dashed line) and for the 100 detected BCGs in the enlarged sample (solid line). The dash-dotted line shows the histogram for the upper limits for the 37 undetected BCGs listed in Table 3. A Hubble constant of $75 \mathrm{~km} \mathrm{~s}^{-1} \mathrm{Mpc}^{-1}$ has been adopted

the statistical sample, confirming again that there is no selection effect in the latter due to telescope sensitivities.

From the notes to Tables 1 and 2 we can estimate that at least $\sim 35 \%$ of the BCGs in the statistical sample show evidence for being member of a pair, a group, or for signs of tidal interaction with another galaxy. This percentage is similar to that found by Campos-Aguilar \& Moles (1991) and Campos-Aguilar et al. (1993) for other BCG samples.

Acknowledgements. The Lyon-Meudon Extragalactic Database was very useful for the confusion analysis of our data and we acknowledge the efforts of the team of people who created and manage it. S.A.P. thanks the ESO C\& EE program for partial financial support through Travel Grant No. B-02-047. T.X.T. has been partially supported by NSF grant AST-9616863, a visiting professorship at the University of Paris VII and the Centre National de la Recherche Scientifique. This collaboration was made possible by NATO collaborative research grant No. 921285. T.X.T. and S.A.P. are grateful to Dr. Chantal Balkowski for her hospitality at the Département d'Astronomie Extragalactique et de Cosmologie at the Observatoire de Paris. Finally, we thank the directors of the Nançay and Green Bank Observatories for generous allotments of observing time which allowed us to carry this project to completion. We thank Claude Carignan for useful comments on the manuscript.

\section{Appendix A: Calibration of the Nançay data}

Since at Nançay, all 9, 18 and 21-cm receivers are permanently installed in the carriage chariot, the noise diode measurements are not made in the laboratory, but are 
Table 4. Comparison with other authors

\begin{tabular}{ccc}
\hline \hline IAU name & \multicolumn{2}{c}{$\begin{array}{c}\text { Integrated flux densities } \\
\left(\mathrm{Jy} \mathrm{km} \mathrm{s}^{-1}\right)\end{array}$} \\
\cline { 2 - 3 } & Other Authors & This Work \\
1 & 2 & 3 \\
\hline $0034+356$ & 1.56 & $1.96 \pm 0.24$ \\
$0143+346$ & 3.11 & $1.99 \pm 0.20$ \\
$0834+518$ & 25.52 & $22.24 \pm 0.48$ \\
$0930+554$ & 2.85 & $2.71 \pm 0.14$ \\
$0940+662$ & 2.03 & $2.86 \pm 1.24$ \\
$0946+558$ & 1.25 & $2.19 \pm 0.90$ \\
$1001+555$ & 4.23 & $4.54 \pm 0.73$ \\
$1123+644$ & 5.26 & $7.52 \pm 1.24$ \\
$1332+518$ & 5.28 & $4.98 \pm 0.53$ \\
$1535+554$ & 1.77 & $1.41 \pm 0.26$ \\
$2127+021$ & 2.03 & $2.00 \pm 0.12$ \\
$2214+162$ & 2.24 & $1.63 \pm 0.22$ \\
$2220+377$ & 7.82 & $8.89 \pm 0.38$ \\
$2233+200$ & 5.28 & $4.47 \pm 0.37$ \\
$2246+315$ & 3.38 & $2.32 \pm 0.27$ \\
\hline
\end{tabular}

made through drift scan observations of standard radiocontinuum sources. Full sets of noise diode temperatures are measured twice a year for many observation frequencies and polarizations, on several extragalactic radio-continuum sources.

Calibration measurements were made at three frequencies: 1370, 1400 and $1425 \mathrm{MHz}$. A general drift of the noise diode temperature as a function of time is seen. The jump occurring on July 24, 1994 (hereafter 940724) is due to a hardware change.

Our observations were performed between November 1992 and mid-1996, but most of the data were taken in 1993 and 1994. The main variations of the noise diode temperature are due to the changing observation frequencies, corresponding to radial velocities of 500$6000 \mathrm{~km} \mathrm{~s}^{-1}$. The following formulas were used: $T_{<940724}=223.3-0.14 \times F$ and $T_{>940724}=63.1-0.032 \times F$ where $T$ is the estimated noise diode temperature in $K$ and $F$ the observation frequency in $\mathrm{MHz}$. The overall calibration procedure accuracy is $\sim 10 \%$.

\section{A1. Comparison with data from other authors}

Among the galaxies which have been detected with a high signal-to-noise ratio in the course of this survey and are not suffering any confusion, 12 have been observed by other authors and their HI parameters can be found in the literature. We have also observed 2 more Markarian galaxies (MK 303 and MK 307) to increase our comparison sample. The comparison sample is given in Table 4, with both our HI measurements and those of other authors.

We have plotted in Fig. 6 our measurements against those of others along with our error bars. The solid line

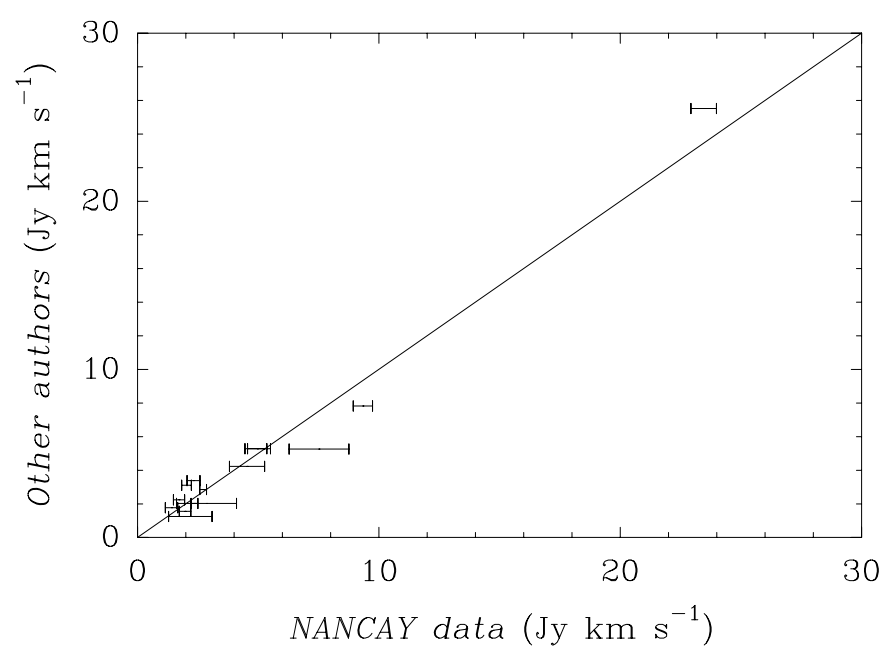

Fig. 6. Integrated HI flux densities from other authors (ordinate axis) versus our Nançay and Green Bank measurements (abscissa axis) for a sample of 15 common galaxies. The solid line represents equality

represents equality. The data points scatter evenly on either side of the equality line, demonstrating the correctness of our flux calibration.

\section{References}

Armstrong J.T., Wootten A., 1986, in Light on Dark Matter: 1st IRAS Conf., Knudsen, p. 439

Bottinelli L., Gouguenheim L., Theureau G., Coudreau N., Paturel G., 1999, A\&AS 135, 429

Campos-Aguilar A., Moles M., 1991, A\&A 241, 358

Campos-Aguilar A., Moles M., Masegosa G., 1993, AJ 106, 1784

Carigi L., Colin P., Peimbert M., Sarmiento A., 1995, ApJ 445, 98

Fanelli M.N., O'Connell R.W., Thuan T.X., 1988, ApJ 334, 665

Garcia A.M., 1993, A\&AS 100, 47

Gordon D., Gottesman S.T., 1981, AJ 86, 161

Haynes M.P., Giovanelli R., Starosta B.M., Magri C., 1988, AJ 95,607

Haynes M.P., Giovanelli R., 1991, ApJS 77, 331

Hickson P., 1982, ApJ 255, 382

Hoffman G.L., Lu N.Y., Salpeter E.E., 1992, AJ 104, 2086

Izotov Yu.I., Thuan T.X., 1998, ApJ 500, 188

Izotov Yu.I., Lipovetsky V.A., Guseva N.G., Kniazev A.Yu., Neizvestny S.I., Stepanian J.A., 1993, Astron. Astroph. Trans. 3, 197

Izotov Yu.I., Lipovetsky V.A., Chaffee F.H., Foltz C.B., Guseva N.G., Kniazev A.Yu., 1997, ApJ 476, 698

Jackson J., Barret A.H., Amstrong J.T., Ho P.T.P., 1987, AJ 93, 531

Keel W.C., van Soest E.T.M., 1992, A\&AS 94, 553

Kniazev A.Yu., 1995 (private communication)

Kraan-Korteweg R.C., 1986, A\&AS 66, 255

Kunth D., Maurogordato S., Vigroux L., 1988, A\&A 204, 10

Lewis B.M., 1987, A\&AS 63, 515 
Lequeux J., Viallefond F., 1980, A\&A 91, 269

Lequeux J., Peimbert M., Rayo J., Serrano A., Torres-Peimbert S., 1979, A\&A 80, 155

Lipovetsky V.A., Chaffee F.H., Izotov Yu.I., Foltz C.B., Kniazev A.Yu., Hopp U., 1999a, ApJ 519, 177

Lipovetsky V.A., Thuan T.X., Richter G.M., Pustilnik S.A., Kniazev A.Yu., Izotov Yu.I., 1999b (in preparation)

Loose H.H., Thuan T.X., 1985, in Star-Forming dwarf galaxies and related objects, Kunth D., Thuan T.X. and Van J.T.T. (eds.). Édition Frontières, p. 73

Marconi G., Matteucci F., Tosi M., 1994, MNRAS 270, 35

Markarian B.E., Lipovetsky V.A., Stepanian J.A., 1983, Afz 19,29

Markarian B.E., Lipovetsky V.A., Stepanian J.A., Erastova L.K., Shapovalova A.I., 1989, Comm. Special Ap. Obs. 62, 5

Mebold U., Goss W.M., van Woerden H., Hawarden T.G., Siegman B., 1979, A\&A 74, 100

Papaderos P., Loose H.H., Thuan T.X., Fricke K.J., 1996, A\&AS 120, 207

Peimbert M., Colin P., Sarmiento A., 1994, in: Violent Star Formation from 30 Doradus to QSOs, Tenorio-Tagle G. (ed.). Cambridge, Cambridge Univ. Press, p. 79

Pesch P., Sanduleak N., 1987, ApJS 63, 809

Pesch P., Sanduleak N., Stephenson C.B., 1991, ApJS 76, 1043

Pilyugin L.S., 1993, A\&A 277, 42

Pustilnik S.A., Ugryumov A.V., Lipovetsky V.A., Thuan T.X., Guseva N.G., 1995, ApJ 443, 499

Pustilnik S.A., Izotov Yu.I., Lipovetsky V.A., Martin J.-M., Thuan T.X., 1996, in: 11-th IAP Colloquium "Interplay between Massive Star Formation, the ISM and Galaxy Evolution", Paris, July 1995, Kunth D., et al. (eds.). Éditions Frontières, p. 585

Pustilnik S.A., Lipovetsky V.A., Izotov Yu.I., Brinks E., Thuan T.X., Kniazev A.Yu., Neizvestny S.I., Ugryumov A.V., 1997, Astron. Lett. 23, 308

Pustilnik S.A., Brinks E., Thuan T.X., Lipovetsky V.A., Izotov Yu.I., 1999 (in preparation)

Salzer J.J., MacAlpine G.M., Boroson T.A., 1989, ApJS 70, 447
Sargent W.L.W., Searle L., 1970, ApJ 162, L155

Schneider S.E., Helou G., Salpeter E.E., Terzian Y., 1986, AJ 92,742

Schneider S.E., Thuan T.X., Magri C., Wadiak J.E., 1990, ApJS 72, 245

Schneider S.E., Thuan T.X., Mangum J.G., Miller J., 1992, ApJS 81, 5

Staveley L., Davies R.D., Kinman T.D., 1992, MNRAS 258, 334

Stepanian J.A., Lipovetsky V.A., Erastova L.K., Shapovalova A.I., Gyulzadian M.B., 1993a, Astrofiz. Issled (Izv. SAO) 35,15

Stepanian J.A., Lipovetsky V.A., Erastova L.K., Shapovalova A.I., Hakopian S.A., 1993b, Astrofiz. Issled (Izv. SAO) 35, 24

Stepanian J.A., Lipovetsky V.A., Erastova L.K., Gyulzadian M.B., Izotov Yu.I., Guseva N.G., 1993c, Astrofiz. Issled (Izv. SAO) 35, 32

Stepanian J.A., Lipovetsky V.A., Erastova L.K., Hakopian S.A., Izotov Yu.I., Guseva N.G., 1993d, Astrofiz. Issled (Izv. SAO) 35, 38

Terlevich R., Melnik J., Masegosa J., Moles M., Copetti M.V.F., 1991, A\&AS 91, 285

Theureau G., Bottinelli L., Coudreau-Durand N., Gouguenheim L., Hallet N., Loulergue M., Paturel G., Teerikorpi P., 1998, A\&AS 130, 333

Thuan T.X., 1983, ApJ 268, 667

Thuan T.X., 1985, ApJ 299, 881

Thuan T.X., 1991, in Massive stars in Starbursts, Leitherer C., Walborn N.R., Heckman T.M. and Norman C.A. (eds.). Cambridge, Cambridge Univ. Press, p. 183

Thuan T.X., Martin G.E., 1981, ApJ 247, 823

Thuan T.X., Izotov Yu.I., 1997, ApJ 489, 623

Thuan T.X., Izotov Yu.I., Lipovetsky V.A., 1995, ApJ 445, 108

Thuan T.X., Izotov Yu.I., Lipovetsky V.A., 1996, ApJ 463, 120

Thuan T.X., Izotov Yu.I., Lipovetsky V.A., 1997, ApJ 477, 661

Viallefond F., Thuan T.X., 1983, ApJ 269, 444

Wegner G., Haynes M.P., Giovannelli R., 1993, AJ 105, 1271 